\title{
Effective collision strengths for transitions in Fe $\mathbf{X}^{\star, \star \star}$
}

\author{
K. M. Aggarwal and F. P. Keenan
}

\begin{abstract}
Department of Physics and Astronomy, Queen's University, Belfast BT7 1NN, Northern Ireland, UK e-mail: K.Aggarwal@qub.ac.uk
\end{abstract}

Received 14 March 2005 / Accepted 10 May 2005

\begin{abstract}
Collision strengths for 4005 transitions among the lowest 90 levels of the $\left(1 s^{2} 2 s^{2} 2 p^{6}\right) 3 s^{2} 3 p^{5}, 3 s 3 p^{6}, 3 s^{2} 3 p^{4} 3 d$, $3 \mathrm{~s} 3 \mathrm{p}^{5} 3 \mathrm{~d}$ and $3 \mathrm{~s}^{2} 3 \mathrm{p}^{3} 3 \mathrm{~d}^{2}$ configurations of Fe $\mathrm{X}$ have been calculated using the Dirac Atomic $R$-matrix Code (DARC) of Norrington \& Grant (2005, Comput. Phys. Commun., in preparation), over a wide energy range up to 210 Ryd. Resonances have been resolved in the threshold region, and effective collision strengths have been obtained over a wide temperature range up to $10^{7} \mathrm{~K}$. The present calculations should represent a significant improvement (in both range and accuracy) over the earlier available results of Bhatia \& Doschek (1995, At. Data Nucl. Data Tables, 60, 97), Tayal (2001, ApJS, 132, 117) and Pelan \& Berrington (2001, A\&A, 365, 258). Based on several comparisons, the accuracy of our data is assessed to be better than $20 \%$, for a majority of transitions.
\end{abstract}

Key words. atomic data - atomic processes

\section{Introduction}

In a recent paper, we (Aggarwal \& Keenan 2004b) reported calculations of energy levels and radiative rates for transitions among the lowest 54 levels of the $\left(1 s^{2} 2 s^{2} 2 p^{6}\right) 3 s^{2} 3 p^{5}$, $3 s 3 p^{6}, 3 s^{2} 3 p^{4} 3 d$ and $3 s 3 p^{5} 3 d$ configurations of Fe $X$, and in this paper we present our results for collision strengths $(\Omega)$ and effective collision strengths $(\Upsilon)$ for transitions among the lowest 90 levels of the $3 s^{2} 3 p^{5}, 3 s 3 p^{6}, 3 s^{2} 3 p^{4} 3 d, 3 s 3 p^{5} 3 d$ and $3 \mathrm{~s}^{2} 3 \mathrm{p}^{3} 3 \mathrm{~d}^{2}$ configurations.

Iron is an abundant element in solar and fusion plasmas, and its emission lines are observed over almost all ionization stages. To analyse the vast amount of observational data available from space missions such as SOHO, Chandra and XMM Newton, theoretical atomic data for $\mathrm{Fe}$ ions are required, because there is paucity of desired experimental results. To this end we have also reported atomic data (namely, energy levels, radiative rates, collision strengths and excitation rate coefficients) for Fe XI (Aggarwal \& Keenan 2003a,b), Fe XIII (Aggarwal \& Keenan 2004a, 2005), Fe XV (Aggarwal et al. 2000, 2001, 2003a), Fe XVII (Aggarwal et al. 2003b, 2004), Fe XVIII (Jonauskas et al. 2004), Fe XXI (Aggarwal $\&$ Keenan 2001), and Fe XXIV (McKeown et al. 2004), and in this paper we present our results of $\Omega$ and $\Upsilon$ for transitions in $\mathrm{Fe} \mathrm{X}$.

Emission lines of Cl-like Fe X in the EUV (150-900 $⿱$ ) and UV (900-2000 $\AA$ ) ranges have been observed in the

\footnotetext{
* Tables 2 and 5 are only available in electronic form at the CDS via anonymous ftp to cdsarc.u-strasbg.fr $(130.79 .128 .5)$ or via http://cdsweb.u-strasbg.fr/cgi-bin/qcat?J/A+A/439/1215

$\star \star$ Figures 6-11 are only available in electronic form at http://www. edpsciences.org
}

spectra of the solar atmosphere (Brosius et al. 1998) and latetype stars (Drake et al. 1995a,b), and have proved to be useful plasma diagnostics (Foster et al. 1996). A famous visible transition of $\mathrm{Fe} \mathrm{X}$ is the red line at $\sim 6375 \AA$ belonging to the $\left(3 \mathrm{~s}^{2} 3 \mathrm{p}^{5}\right){ }^{2} \mathrm{P}_{3 / 2}^{\circ}-{ }^{2} \mathrm{P}_{1 / 2}^{\circ}$ magnetic dipole (M1) transition. Therefore, realising the importance of Fe X, a number of workers, particularly in the past decade, have performed a variety of calculations to compute various atomic parameters. The most notable among the available data, particularly for $\Omega$ and $\Upsilon$, are the calculations of Bhatia \& Doschek (1995, henceforth BD), Tayal (2000, 2001) and Pelan \& Berrington (2001). However, we find that there is scope for improvement as well as an extension of the available data. We discuss this below.

BD have reported results for energy levels, radiative rates ( $A$-values) and collision strengths for all transitions among 54 levels of the $3 s^{2} 3 p^{5}, 3 s 3 p^{6}, 3 s^{2} 3 p^{4} 3 d$ and $3 s 3 p^{5} 3 d$ configurations. They have adopted the SuperStructure (SS) code of Eissner et al. (1974) for the generation of wavefunctions, and the Distorted-Wave (DW) code of Eissner \& Seaton (1972) for the computation of $\Omega$. Their calculations are basically in $L S$ coupling, and results for transitions in intermediate coupling $(L S J)$ were obtained through an algebraic transformation using the JAJOM program of Saraph (1972). One-body relativistic effects were included in the calculations through term coupling coefficients. However, their calculations are limited mainly for two reasons. Firstly, their wavefunctions are not accurate, because many of their energy levels differ with experimental and other theoretical results by $\sim 5 \%$, and some by up to $11 \%$ - see Table 1 of Aggarwal \& Keenan (2004b). This has a direct effect on the subsequent calculations of radiative rates and collision strengths, which may easily vary by a factor of $\sim 2$ for strong transitions, and by larger factors for weaker 
transitions - see, for example, Tables 3 and 4 of Tayal (2001). The main reason for this inaccuracy is the neglect of configuration interaction $(\mathrm{CI})$ with additional configurations, as $\mathrm{CI}$ is very important for Fe $\mathrm{X}$. This is in spite of it being a moderately heavy ion $(Z=26)$, as already explained and demonstrated in our earlier paper (Aggarwal \& Keenan 2004b). Secondly, and more importantly, BD have reported values of $\Omega$ at only 5 energies (9-45 Ryd) above thresholds, and have not resolved resonances in the thresholds region. Therefore, any results obtained for $\Upsilon$ from their limited data of $\Omega$ will be highly underestimated, especially for the forbidden transitions, because resonances may enhance values of $\Upsilon$ up to an order of magnitude, depending on the transition and the temperature.

Tayal (2001, henceforth SST) attempted to remove both the limitations of the calculations by BD. For the generation of wavefunctions, he included additional CI (mainly with the $4 \ell$ orbitals), and resolved resonances in order to improve the accuracy of the $\Upsilon$ results. Furthermore, he adopted an independent approach by employing the CIV3 program of Hibbert (1975) for the generation of wavefunctions, and the $R$-matrix code of Berrington et al. (1995) for the computation of $\Omega$. His calculations were performed in LSJ coupling, and one-body relativistic effects were included through the Breit-Pauli approximation. However, his calculations are also limited for three main reasons. Firstly, his lowest 24 energy levels are the highest among all the available theoretical results, and are higher by up to $8 \%$ in comparison with the experimental ones - see, for example, levels 19 and 20 in Table 1 of SST. Additionally, the orderings of his energy levels agree neither with the experimental ones nor with any of the CI calculations of Pelan \& Berrington (2001), Deb et al. (2002), or Aggarwal \& Keenan (2004b). Secondly, SST excluded 10 levels, namely $3 s^{2} 3 p^{4}\left({ }^{1} \mathrm{D}\right) 3 \mathrm{~d}$ ${ }^{2} \mathrm{G}_{7 / 2,9 / 2}, 3 \mathrm{~s} 3 \mathrm{p}^{5}\left({ }^{3} \mathrm{P}\right) 3 \mathrm{~d}{ }^{4} \mathrm{~F}_{3 / 2,5 / 2,7 / 2,9 / 2}^{\circ}, 3 \mathrm{~s} 3 \mathrm{p}^{5}\left({ }^{3} \mathrm{P}\right) 3 \mathrm{~d}^{2} \mathrm{~F}_{5 / 2,7 / 2}^{\circ}$ and $3 \mathrm{~s} 3 \mathrm{p}^{5}\left({ }^{1} \mathrm{P}\right) 3 \mathrm{~d}^{2} \mathrm{~F}_{5 / 2,7 / 2}^{\circ}$. These metastable levels are spectroscopically important (Del Zanna et al. 2004), and therefore results for transitions involving these levels are not only required but their omissions also affect the resonance patterns in the thresholds region, and subsequently their contributions in the determination of $\Upsilon$ values. Finally, and most importantly, he reported results of $\Upsilon$ only for transitions from the lowest three levels to higher excited levels. However, results for all transitions are required for a diagnostic or modelling calculation, and this has been clearly emphasized and demonstrated by Del Zanna et al. - see particularly their Fig. 2 and Table 6.

Finally, there are calculations by Pelan \& Berrington (2001, henceforth PB), who have adopted a similar approach to SST, i.e. they too have performed their calculations in $L S J$ coupling through the Breit-Pauli approximation, and have employed the CIV3 and $R$-matrix programs for the generation of wavefunctions and computations of $\Omega$, respectively. They have included extensive $\mathrm{CI}$ in the generation of wavefunctions, which is clearly reflected by the excellent agreement their energy levels have, not only in magnitude but also in ordering, with the experimental ones and with other theoretical results of Deb et al. (2002) and Aggarwal \& Keenan (2004b). Additionally, they too have resolved resonances in a fine energy mesh, and hence their results for all atomic parameters should be comparatively more accurate than those of BD or SST. However, their results are limited to transitions from the lowest three levels to higher excited levels, but in a recent paper (Del Zanna et al. 2004) they have widened the range by also reporting results for the remaining 378 transitions. Nevertheless, their results for $\Upsilon$ are still confined to the lowest 31 levels of the $3 s^{2} 3 p^{5}, 3 s 3 p^{6}$ and $3 s^{2} 3 p^{4} 3 d$ configurations, whereas corresponding results for transitions involving the additional 23 levels of the $3 \mathrm{~s} 3 \mathrm{p}^{5} 3 \mathrm{~d}$ configuration are also required, as has been emphasized by Del Zanna et al., because transitions involving these levels have already been observed in solar plasmas. Apart from this limitation of their work, there is some concern about the accuracy of their results, which we discuss below.

The PB results for $\Upsilon$ differ with those of SST by a factor of two for many transitions, such as $2-3,4,5,6,7-$ see Table 1 for level indices. These substantial differences are not confined to any particular range of temperature, but are noticeable for the entire range $\left(5.4 \leq \log T_{\mathrm{e}}(\mathrm{K}) \leq 6.8\right)$ for some of the transitions, such as $2-4$ and $2-5$. Similarly, their values of $\Omega$ differ by a factor of two for some of the allowed transitions, such as $1-3$ and $2-3$. This is in spite of the fact that the transition energies and oscillator strengths ( $f$-values) are comparable in both calculations. Since both of these calculations (of SST and PB) are contemporary, are of comparable accuracy, and adopt the same methodology (of using the $R$-matrix code in the Breit-Pauli approximation), such large differences for many transitions of importance are not expected. Therefore, our aim is to assess the accuracy of the available data for $\Omega$ and $\Upsilon$, as well as to extend the range of transitions. We achieve this aim by performing a calculation by an independent approach. Contrary to the already available calculations, our approach is fully relativistic as we employ the GRASP (General purpose Relativistic Atomic Structure Package) code of Dyall et al. (1989) for the generation of wavefunctions, and the Dirac Atomic $R$-matrix Code (DARC) of Norrington \& Grant (2005) for the computations of $\Omega$, and subsequently of $\Upsilon$.

\section{Atomic structure}

The $3 s^{2} 3 p^{5}, 3 s 3 p^{6}, 3 s^{2} 3 p^{4} 3 d$ and $3 s 3 p^{5} 3 d$ configurations of $\mathrm{Fe} \mathrm{X}$ give rise to 54 fine-structure levels. However, energy levels obtained with CI among these four configurations alone differ by up to $5 \%$ when compared with the corresponding experimental results compiled by NIST (http://physics.nist.gov/PhysRefData) - see Table 1 of Aggarwal \& Keenan (2004b). Since experimental energies for the higher levels of the $3 \mathrm{~s}^{2} 3 \mathrm{p}^{3} 3 \mathrm{~d}^{2}$ configuration are not yet available, no direct comparisons can be made to assess the accuracy of the theoretical results. However, the importance of the inclusion of larger CI in the accurate determination of energy levels has already been emphasized and demonstrated by Pelan \& Berrington (2001), Deb et al. (2002), and Aggarwal \& Keenan (2004b). Energy levels obtained with limited CI (among the above stated 4 configurations) may differ by up to $11 \%$ in comparison with those obtained with extensive CI - see Table 1 of Aggarwal \& Keenan. Therefore, based on several test calculations, we concluded in our earlier paper 
(Aggarwal \& Keenan 2004b) that to achieve reasonable accuracy in energy levels and radiative rates, CI among 8 configurations (namely, $3 s^{2} 3 p^{5}, 3 s 3 p^{6}, 3 s^{2} 3 p^{4} 3 d, 3 s 3 p^{5} 3 d, 3 p^{6} 3 d$, $3 s 3 p^{4} 3 d^{2}, 3 s^{2} 3 p^{3} 3 d^{2}$ and $\left.3 s^{2} 3 p^{2} 3 d^{3}\right)$ is necessary. However, these configurations give rise to 669 levels, with which a scattering calculation cannot be performed with the resources available to us. Therefore, a compromise has to be made. Since 36 levels of the $3 \mathrm{~s}^{2} 3 \mathrm{p}^{3} 3 \mathrm{~d}^{2}$ configuration lie below the highest level ${ }^{2} \mathrm{P}_{3 / 2}^{\circ}$ of the $3 \mathrm{~s} 3 \mathrm{p}^{5} 3 \mathrm{~d}$ configuration, their inclusion in the scattering calculation becomes necessary. Therefore, in the present work we have included the lowest 90 levels of the $3 s^{2} 3 p^{5}, 3 s 3 p^{6}, 3 s^{2} 3 p^{4} 3 d, 3 s 3 p^{5} 3 d$ and $3 s^{2} 3 p^{3} 3 d^{2}$ configurations of $\mathrm{Fe} \mathrm{X}$. The inclusion of additional levels will not only improve the results for effective collision strengths, but may also be beneficial to spectral modelling.

To generate the wavefunctions, we have used the fully relativistic GRASP code of Dyall et al. (1989) with the option of extended average level (EAL), in which a weighted (proportional to $2 j+1$ ) trace of the Hamiltonian matrix is minimized. This produces a compromise set of orbitals describing closely lying states with moderate accuracy. Our calculations are in the $j j$ coupling scheme, and Breit and QED corrections have been included. CI has been included among the above stated eight configurations, and energy levels obtained are the same as reported in Table 1 of Aggarwal \& Keenan (2004b) under the column GRASP8. However, in that paper we reported energies for the 54 levels of the lowest four configurations only, and here in Table 1 we list energies for the lowest 90 levels among the $3 s^{2} 3 p^{5}, 3 s 3 p^{6}, 3 s^{2} 3 p^{4} 3 d, 3 s 3 p^{5} 3 d$ and $3 s^{2} 3 p^{3} 3 d^{2}$ configurations. Since an elaborate comparison has already been made of our energies with other available theoretical and experimental results, and no corresponding data are available in the literature for the additional higher lying 36 levels now included, we do not discuss our energy levels any further. However, based on several comparisons, the accuracy of our energy levels is assessed to be better than 3\%. Similarly, in our earlier paper we reported $A$-values for (E1, E2, M1 and M2) transitions among the 54 levels of the $3 s^{2} 3 p^{5}, 3 s 3 p^{6}, 3 s^{2} 3 p^{4} 3 d$ and $3 s 3 p^{5} 3 d$ configurations, and corresponding results involving the higher levels of the $3 \mathrm{~s}^{2} 3 \mathrm{p}^{3} 3 \mathrm{~d}^{2}$ configuration can be obtained on request from one of the authors (K. Aggarwal@qub.ac.uk).

\section{Collision strengths}

For the computations of collision strengths, we have employed the DARC program of Norrington \& Grant (2005). This program includes the relativistic effects in a systematic way, in both the target description and the scattering model. It is based on the $j j$ coupling scheme, and uses the Dirac-Coulomb Hamiltonian in the $R$-matrix approach. However, because of the inclusion of fine-structure in the definition of channel coupling, the matrix size of the Hamiltonian increases substantially, making the calculations computationally more expensive. The $R$-matrix radius has been adopted to be 3.28 au, and 21 continuum orbitals have been included for each channel angular momentum for the expansion of the wavefunction. This allows us to compute $\Omega$ up to an energy of 210 Ryd. The maximum number of channels for a partial wave is 538 , and

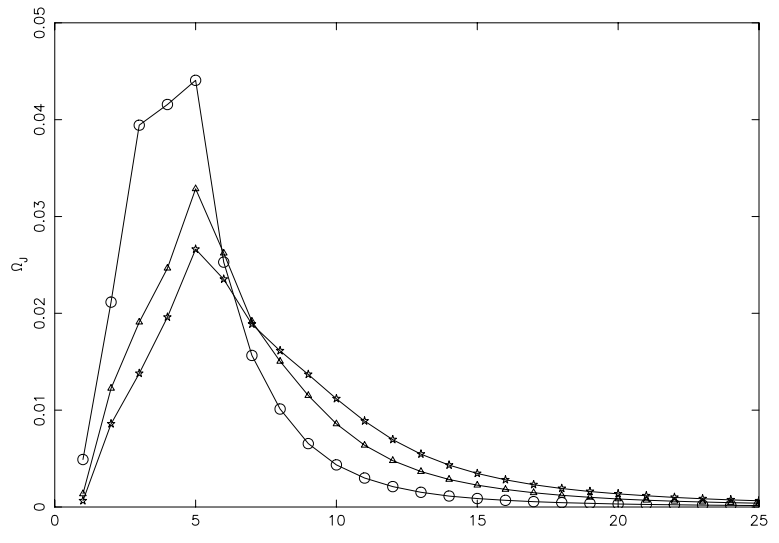

(a)

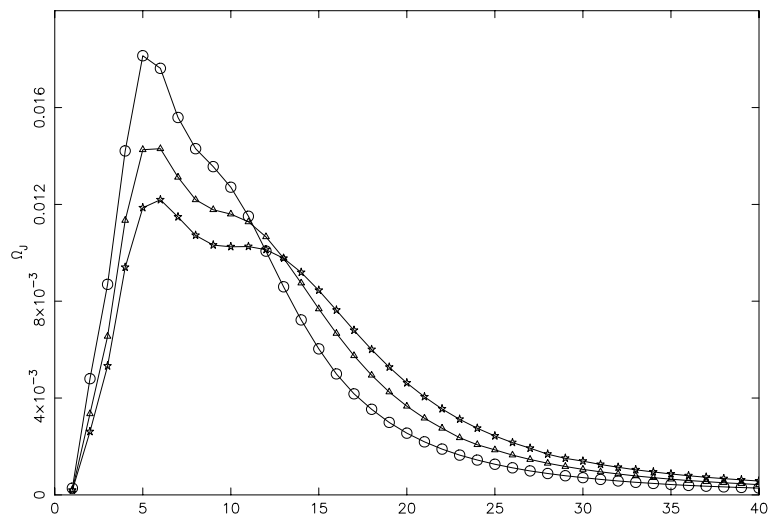

(b)

Fig. 1. Partial collision strengths for the $\left(3 s^{2} 3 p^{5}\right){ }^{2} \mathrm{P}_{3 / 2}^{\circ}-{ }^{2} \mathrm{P}_{1 / 2}^{\circ}$ (1-2) transition of $\mathrm{Fe} \mathrm{X}$, at three energies of: a) $10 \mathrm{Ryd}$ (circles), 30 Ryd (triangles), and 50 Ryd (stars), and b) 100 Ryd (circles), 150 Ryd (triangles), and 200 Ryd (stars).

the corresponding size of the Hamiltonian matrix is 11340 . In order to obtain convergence of $\Omega$ for all transitions and at all energies, we have included all partial waves with angular momentum $J \leq 39$, although a higher range would have been preferable for the convergence of allowed transitions, especially at higher energies. However, given the resources available to us, this was not possible. Nevertheless, to account for the inclusion of higher neglected partial waves, we have included a top-up, based on the Coulomb-Bethe approximation for allowed transitions and geometric series for forbidden transitions. We will further elaborate about it later in this section.

In Figs. 1a, 2a and 3a we show the variation of $\Omega$ with angular momentum $J$ at three energies of 10 , 30 and $50 \mathrm{Ryd}$, and for three transitions, namely 1-2 $\left(3 s^{2} 3 p^{5}{ }^{2} \mathrm{P}_{3 / 2}^{\circ}-3 s^{2} 3 p^{5}{ }^{2} \mathrm{P}_{1 / 2}^{\circ}\right), 1-3\left(3 s^{2} 3 p^{5}{ }^{2} \mathrm{P}_{3 / 2}^{\circ}-3 s 3 p^{6}{ }^{2} \mathrm{~S}_{1 / 2}\right)$ and $3-26\left(3 s 3 p^{6}{ }^{2} S_{1 / 2}-3 s^{2} 3 p^{4}\left({ }^{1} S\right) 3 d{ }^{2} D_{5 / 2}\right)$, which are parity forbidden, allowed, and forbidden, respectively. The corresponding results at three higher energies of 100, 150 and 200 Ryd are shown in Figs. 1b, $2 b$ and 3b, respectively. For the forbidden transitions, such as 1-2 and 3-26 shown in Figs. 1 ( $a$ and b) and 3 ( $a$ and b), $\Omega$ have (almost) fully converged at all energies, including the highest energy of our calculations, i.e. 210 Ryd. For allowed transitions, such 
Table 1. Target levels of Fe X and their threshold energies (in Ryd).

\begin{tabular}{|c|c|c|c|c|c|c|c|c|c|}
\hline Index & Configuration & Level & $\operatorname{GRASP}^{a}$ & GRASP $^{b}$ & Index & Configuration & Level & $\operatorname{GRASP}^{a}$ & GRASP $^{b}$ \\
\hline $1 \ldots \ldots$ & $3 s^{2} 3 p^{5}$ & ${ }^{2} \mathrm{P}_{3 / 2}^{\circ}$ & 0.0000 & 0.0000 & $46 \ldots . .$. & $3 s 3 p^{5}\left({ }^{3} P\right) 3 d$ & ${ }^{2} \mathrm{D}_{5 / 2}^{\circ}$ & 7.0780 & 7.0664 \\
\hline $2 \ldots \ldots$ & $3 s^{2} 3 p^{5}$ & ${ }^{2} \mathrm{P}_{1 / 2}^{\circ}$ & 0.1461 & 0.1426 & $47 \ldots \ldots$ & $3 \mathrm{~s} 3 \mathrm{p}^{5}\left({ }^{1} \mathrm{P}\right) 3 \mathrm{~d}$ & ${ }^{2} \mathrm{P}_{1 / 2}^{\circ}$ & 7.1552 & 7.1458 \\
\hline $3 \ldots \ldots$ & $3 s 3 p^{6}$ & ${ }^{2} \mathrm{~S}_{1 / 2}$ & 2.6066 & 2.6024 & $48 \ldots \ldots$ & $3 \mathrm{~s} 3 \mathrm{p}^{5}\left({ }^{1} \mathrm{P}\right) 3 \mathrm{~d}$ & ${ }^{2} \mathrm{P}_{3 / 2}^{\circ}$ & 7.2331 & 7.2206 \\
\hline $4 \ldots \ldots$ & $3 s^{2} 3 p^{4}\left({ }^{3} P\right) 3 d$ & ${ }^{4} \mathrm{D}_{5 / 2}$ & 3.5545 & 3.5473 & $49 \ldots \ldots$ & $3 s^{2} 3 p^{3}\left({ }^{4} S\right) 3 d^{2}\left({ }^{3} F\right)$ & ${ }^{6} \mathrm{~F}_{1 / 2}^{\circ}$ & 7.2842 & 7.2722 \\
\hline$\star 5 \ldots \ldots$ & $3 s^{2} 3 p^{4}\left({ }^{3} P\right) 3 d$ & ${ }^{4} \mathrm{D}_{7 / 2}$ & 3.5550 & 3.5468 & 50. & $3 s^{2} 3 p^{3}\left({ }^{4} S\right) 3 d^{2}\left({ }^{3} F\right)$ & ${ }^{6} \mathrm{~F}_{3 / 2}^{\circ}$ & 7.2859 & 7.2736 \\
\hline $6 \ldots \ldots$ & $3 s^{2} 3 p^{4}\left({ }^{3} P\right) 3 d$ & ${ }^{4} \mathrm{D}_{3 / 2}$ & 3.5653 & 3.5587 & $51 \ldots \ldots$ & $3 s^{2} 3 p^{3}\left({ }^{4} S\right) 3 d^{2}\left({ }^{3} F\right)$ & ${ }^{6} \mathrm{~F}_{5 / 2}^{\circ}$ & 7.2892 & 7.2762 \\
\hline $7 \ldots \ldots$ & $3 s^{2} 3 p^{4}\left({ }^{3} P\right) 3 d$ & ${ }^{4} \mathrm{D}_{1 / 2}$ & 3.5791 & 3.5724 & $52 \ldots \ldots$ & $3 s^{2} 3 p^{3}\left({ }^{4} S\right) 3 d^{2}\left({ }^{3} F\right)$ & ${ }^{6} \mathrm{~F}_{7 / 2}^{\circ}$ & 7.2942 & 7.2803 \\
\hline $8 \ldots \ldots$ & $3 s^{2} 3 p^{4}\left({ }^{3} P\right) 3 d$ & ${ }^{4} \mathrm{~F}_{9 / 2}$ & 3.8485 & 3.8407 & $53 \ldots \ldots$ & $3 s^{2} 3 p^{3}\left({ }^{4} S\right) 3 d^{2}\left({ }^{3} F\right)$ & ${ }^{6} \mathrm{~F}_{9 / 2}^{\circ}$ & 7.3012 & 7.2862 \\
\hline $9 \ldots \ldots$ & $3 s^{2} 3 p^{4}\left({ }^{1} D\right) 3 d$ & ${ }^{2} \mathrm{P}_{1 / 2}$ & 3.8673 & 3.8608 & $54 \ldots \ldots$ & $3 s^{2} 3 p^{3}\left({ }^{4} S\right) 3 d^{2}\left({ }^{3} F\right)$ & ${ }^{6} \mathrm{~F}_{11 / 2}^{\circ}$ & 7.3101 & 7.2935 \\
\hline $10 \ldots \ldots$ & $3 s^{2} 3 p^{4}\left({ }^{3} P\right) 3 d$ & ${ }^{4} \mathrm{~F}_{7 / 2}$ & 3.8954 & 3.8876 & $55 \ldots \ldots$ & $3 \mathrm{~s} 3 \mathrm{p}^{5}\left({ }^{1} \mathrm{P}\right) 3 \mathrm{~d}$ & ${ }^{2} \mathrm{~F}_{5 / 2}^{\circ}$ & 7.4346 & 7.4237 \\
\hline $11 \ldots \ldots$ & $3 s^{2} 3 p^{4}\left({ }^{3} P\right) 3 d$ & ${ }^{4} \mathrm{~F}_{5 / 2}$ & 3.9301 & 3.9220 & $56 \ldots \ldots$ & $3 \mathrm{~s} 3 \mathrm{p}^{5}\left({ }^{1} \mathrm{P}\right) 3 \mathrm{~d}$ & ${ }^{2} \mathrm{~F}_{7 / 2}^{\circ}$ & 7.4756 & 7.4626 \\
\hline $12 \ldots \ldots$ & $3 s^{2} 3 p^{4}\left({ }^{3} P\right) 3 d$ & ${ }^{4} \mathrm{~F}_{3 / 2}$ & 3.9421 & 3.9345 & $57 \ldots \ldots$ & $3 s^{2} 3 p^{3}\left({ }^{4} S\right) 3 d^{2}\left({ }^{3} P\right)$ & ${ }^{6} \mathrm{P}_{3 / 2}^{\circ}$ & 7.5391 & 7.5263 \\
\hline $13 \ldots \ldots$ & $3 s^{2} 3 p^{4}\left({ }^{1} D\right) 3 d$ & ${ }^{2} \mathrm{P}_{3 / 2}$ & 3.9451 & 3.9375 & $58 \ldots \ldots$ & $3 s^{2} 3 p^{3}\left({ }^{4} S\right) 3 d^{2}\left({ }^{3} P\right)$ & ${ }^{6} \mathrm{P}_{5 / 2}^{\circ}$ & 7.5427 & 7.5295 \\
\hline $14 \ldots \ldots$ & $3 s^{2} 3 p^{4}\left({ }^{1} D\right) 3 d$ & ${ }^{2} \mathrm{D}_{3 / 2}$ & 4.0010 & 3.9942 & $59 \ldots \ldots$ & $3 s^{2} 3 p^{3}\left({ }^{4} S\right) 3 d^{2}\left({ }^{3} P\right)$ & ${ }^{6} \mathrm{P}_{7 / 2}^{\circ}$ & 7.5470 & 7.5323 \\
\hline $15 \ldots \ldots$ & $3 s^{2} 3 p^{4}\left({ }^{3} P\right) 3 d$ & ${ }^{4} \mathrm{P}_{1 / 2}$ & 4.0050 & 3.9992 & $60 \ldots \ldots$ & $3 \mathrm{~s} 3 \mathrm{p}^{5}\left({ }^{1} \mathrm{P}\right) 3 \mathrm{~d}$ & ${ }^{2} \mathrm{D}_{3 / 2}^{\circ}$ & 7.6883 & 7.6777 \\
\hline $16 \ldots \ldots$ & $3 s^{2} 3 p^{4}\left({ }^{3} P\right) 3 d$ & ${ }^{4} \mathrm{P}_{3 / 2}$ & 4.0516 & 4.0435 & $61 \ldots \ldots$ & $3 \mathrm{~s} 3 \mathrm{p}^{5}\left({ }^{1} \mathrm{P}\right) 3 \mathrm{~d}$ & ${ }^{2} \mathrm{D}_{5 / 2}^{\circ}$ & 7.7055 & 7.6931 \\
\hline $17 \ldots \ldots$ & $3 s^{2} 3 p^{4}\left({ }^{3} P\right) 3 d$ & ${ }^{4} \mathrm{P}_{5 / 2}$ & 4.0700 & 4.0615 & $62 \ldots$ & $3 s^{2} 3 p^{3}\left({ }^{4} S\right) 3 d^{2}\left({ }^{1} D\right)$ & ${ }^{4} \mathrm{D}_{7 / 2}^{\circ}$ & 7.7747 & 7.7604 \\
\hline $18 \ldots \ldots$ & $3 s^{2} 3 p^{4}\left({ }^{3} P\right) 3 d$ & ${ }^{2} \mathrm{~F}_{7 / 2}$ & 4.0785 & 4.0716 & $63 \ldots \ldots$ & $3 s^{2} 3 p^{3}\left({ }^{4} S\right) 3 d^{2}\left({ }^{1} D\right)$ & ${ }^{4} \mathrm{D}_{5 / 2}^{\circ}$ & 7.7815 & 7.7680 \\
\hline $19 \ldots \ldots$ & $3 s^{2} 3 p^{4}\left({ }^{1} D\right) 3 d$ & ${ }^{2} \mathrm{D}_{5 / 2}$ & 4.0932 & 4.0836 & $64 \ldots \ldots$ & $3 s^{2} 3 p^{3}\left({ }^{4} S\right) 3 d^{2}\left({ }^{1} D\right)$ & ${ }^{4} \mathrm{D}_{3 / 2}^{\circ}$ & 7.7860 & 7.7734 \\
\hline $20 \ldots \ldots$ & $3 s^{2} 3 p^{4}\left({ }^{1} D\right) 3 d$ & ${ }^{2} \mathrm{G}_{9 / 2}$ & 4.1716 & 4.1612 & $65 \ldots \ldots$ & $3 s^{2} 3 p^{3}\left({ }^{4} S\right) 3 d^{2}\left({ }^{1} D\right)$ & ${ }^{4} \mathrm{D}_{1 / 2}^{\circ}$ & 7.7977 & 7.7850 \\
\hline $21 \ldots \ldots$ & $3 s^{2} 3 p^{4}\left({ }^{1} D\right) 3 d$ & ${ }^{2} G_{7 / 2}$ & 4.1750 & 4.1660 & $66 \ldots \ldots$ & $3 s^{2} 3 p^{3}\left({ }^{2} D\right) 3 d^{2}\left({ }^{3} F\right)$ & ${ }^{4} \mathrm{G}_{5 / 2}^{\circ}$ & 7.8071 & 7.7946 \\
\hline $22 \ldots \ldots$ & $3 s^{2} 3 p^{4}\left({ }^{3} P\right) 3 d$ & ${ }^{2} \mathrm{~F}_{5 / 2}$ & 4.2027 & 4.1935 & $67 \ldots \ldots$ & $3 s^{2} 3 p^{3}\left({ }^{2} \mathrm{D}\right) 3 d^{2}\left({ }^{3} \mathrm{P}\right)$ & ${ }^{4} \mathrm{P}_{1 / 2}^{\circ}$ & 7.8082 & 7.7951 \\
\hline $23 \ldots \ldots$ & $3 s^{2} 3 p^{4}\left({ }^{1} D\right) 3 d$ & ${ }^{2} \mathrm{~F}_{5 / 2}$ & 4.4736 & 4.4664 & $68 \ldots \ldots$ & $3 \mathrm{~s}^{2} 3 \mathrm{p}^{3}\left({ }^{2} \mathrm{D}\right) 3 \mathrm{~d}^{2}\left({ }^{3} \mathrm{~F}\right)$ & ${ }^{4} \mathrm{G}_{7 / 2}^{\circ}$ & 7.8179 & 7.8046 \\
\hline $24 \ldots \ldots$ & $3 s^{2} 3 p^{4}\left({ }^{1} D\right) 3 d$ & ${ }^{2} \mathrm{~F}_{7 / 2}$ & 4.5114 & 4.5020 & $69 \ldots \ldots$ & $3 s^{2} 3 p^{3}\left({ }^{2} \mathrm{D}\right) 3 d^{2}\left({ }^{3} \mathrm{P}\right)$ & ${ }^{4} \mathrm{~F}_{3 / 2}^{\circ}$ & 7.8248 & 7.8127 \\
\hline $25 \ldots \ldots$ & $3 \mathrm{~s}^{2} 3 \mathrm{p}^{4}\left({ }^{1} \mathrm{~S}\right) 3 \mathrm{~d}$ & ${ }^{2} \mathrm{D}_{3 / 2}$ & 4.7315 & 4.7243 & $70 \ldots \ldots$ & $3 \mathrm{~s}^{2} 3 \mathrm{p}^{3}\left({ }^{2} \mathrm{D}\right) 3 \mathrm{~d}^{2}\left({ }^{3} \mathrm{~F}\right)$ & ${ }^{4} \mathrm{G}_{9 / 2}^{\circ}$ & 7.8380 & 7.8230 \\
\hline $26 \ldots \ldots$ & $3 s^{2} 3 p^{4}\left({ }^{1} S\right) 3 d$ & ${ }^{2} \mathrm{D}_{5 / 2}$ & 4.7715 & 4.7618 & $71 \ldots \ldots$ & $3 \mathrm{~s}^{2} 3 \mathrm{p}^{3}\left({ }^{2} \mathrm{D}\right) 3 \mathrm{~d}^{2}\left({ }^{3} \mathrm{P}\right)$ & ${ }^{4} \mathrm{P}_{3 / 2}^{\circ}$ & 7.8407 & 7.8272 \\
\hline $27 \ldots \ldots$ & $3 \mathrm{~s}^{2} 3 \mathrm{p}^{4}\left({ }^{1} \mathrm{D}\right) 3 \mathrm{~d}$ & ${ }^{2} S_{1 / 2}$ & 5.0297 & 5.0228 & $72 \ldots \ldots$ & $3 \mathrm{~s}^{2} 3 \mathrm{p}^{3}\left({ }^{2} \mathrm{D}\right) 3 \mathrm{~d}^{2}\left({ }^{3} \mathrm{P}\right)$ & ${ }^{4} \mathrm{~F}_{5 / 2}^{\circ}$ & 7.8590 & 7.8452 \\
\hline $28 \ldots \ldots$ & $3 s^{2} 3 p^{4}\left({ }^{3} P\right) 3 d$ & ${ }^{2} \mathrm{P}_{3 / 2}$ & 5.2800 & 5.2727 & $\star 73 \ldots \ldots$ & $3 \mathrm{~s}^{2} 3 \mathrm{p}^{3}\left({ }^{2} \mathrm{D}\right) 3 \mathrm{~d}^{2}\left({ }^{3} \mathrm{~F}\right)$ & ${ }^{4} \mathrm{G}_{11 / 2}^{\circ}$ & 7.8593 & 7.8426 \\
\hline $29 \ldots \ldots$ & $3 s^{2} 3 p^{4}\left({ }^{3} P\right) 3 d$ & ${ }^{2} \mathrm{P}_{1 / 2}$ & 5.3332 & 5.3243 & $74 \ldots \ldots$ & $3 \mathrm{~s}^{2} 3 \mathrm{p}^{3}\left({ }^{2} \mathrm{D}\right) 3 \mathrm{~d}^{2}\left({ }^{3} \mathrm{P}\right)$ & ${ }^{4} \mathrm{P}_{5 / 2}^{\circ}$ & 7.8736 & 7.8591 \\
\hline $30 \ldots \ldots$ & $3 s^{2} 3 p^{4}\left({ }^{3} P\right) 3 d$ & ${ }^{2} \mathrm{D}_{5 / 2}$ & 5.3503 & 5.3428 & $75 \ldots \ldots$ & $3 \mathrm{~s}^{2} 3 \mathrm{p}^{3}\left({ }^{2} \mathrm{D}\right) 3 \mathrm{~d}^{2}\left({ }^{3} \mathrm{P}\right)$ & ${ }^{4} \mathrm{~F}_{7 / 2}^{\circ}$ & 7.8963 & 7.8813 \\
\hline $31 \ldots \ldots$ & $3 s^{2} 3 p^{4}\left({ }^{3} P\right) 3 d$ & ${ }^{2} \mathrm{D}_{3 / 2}$ & 5.4739 & 5.4633 & $76 \ldots \ldots$ & $3 \mathrm{~s}^{2} 3 \mathrm{p}^{3}\left({ }^{2} \mathrm{D}\right) 3 \mathrm{~d}^{2}\left({ }^{3} \mathrm{P}\right)$ & ${ }^{4} \mathrm{~F}_{9 / 2}^{\circ}$ & 7.9270 & 7.9102 \\
\hline $32 \ldots \ldots$ & $3 s 3 p^{5}\left({ }^{3} P\right) 3 d$ & ${ }^{4} \mathrm{P}_{1 / 2}^{\circ}$ & 6.2330 & 6.2252 & $77 \ldots \ldots$ & $3 \mathrm{~s}^{2} 3 \mathrm{p}^{3}\left({ }^{2} \mathrm{D}\right) 3 \mathrm{~d}^{2}\left({ }^{3} \mathrm{~F}\right)$ & ${ }^{4} \mathrm{H}_{7 / 2}^{\circ}$ & 7.9866 & 7.9746 \\
\hline $33 \ldots \ldots$ & $3 \mathrm{~s} 3 \mathrm{p}^{5}\left({ }^{3} \mathrm{P}\right) 3 \mathrm{~d}$ & ${ }^{4} \mathrm{P}_{3 / 2}^{\circ}$ & 6.2578 & 6.2485 & $78 \ldots \ldots$ & $3 s^{2} 3 p^{3}\left({ }^{2} D\right) 3 d^{2}\left({ }^{3} F\right)$ & ${ }^{4} \mathrm{H}_{9 / 2}^{\circ}$ & 7.9968 & 7.9833 \\
\hline $34 \ldots \ldots$ & $3 s 3 p^{5}\left({ }^{3} P\right) 3 d$ & ${ }^{4} \mathrm{P}_{5 / 2}^{\circ}$ & 6.3016 & 6.2905 & $79 \ldots \ldots$ & $3 s^{2} 3 p^{3}\left({ }^{2} D\right) 3 d^{2}\left({ }^{3} F\right)$ & ${ }^{4} \mathrm{H}_{11 / 2}^{\circ}$ & 8.0118 & 7.9964 \\
\hline $35 \ldots \ldots$ & $3 s 3 p^{5}\left({ }^{3} P\right) 3 d$ & ${ }^{4} \mathrm{~F}_{9 / 2}^{\circ}$ & 6.5623 & 6.5516 & $80 \ldots \ldots$ & $3 s^{2} 3 p^{3}\left({ }^{2} D\right) 3 d^{2}\left({ }^{3} F\right)$ & ${ }^{4} \mathrm{H}_{13 / 2}^{\circ}$ & 8.0378 & 8.0197 \\
\hline $36 \ldots \ldots$ & $3 s 3 p^{5}\left({ }^{3} P\right) 3 d$ & ${ }^{4} \mathrm{~F}_{7 / 2}^{\circ}$ & 6.5873 & 6.5771 & $81 \ldots \ldots$ & $3 s^{2} 3 p^{3}\left({ }^{2} D\right) 3 d^{2}\left({ }^{1} D\right)$ & ${ }^{2} \mathrm{~F}_{7 / 2}^{\circ}$ & 8.0691 & 8.0553 \\
\hline $37 \ldots \ldots$ & $3 \mathrm{~s} 3 \mathrm{p}^{5}\left({ }^{3} \mathrm{P}\right) 3 \mathrm{~d}$ & ${ }^{4} \mathrm{~F}_{5 / 2}^{\circ}$ & 6.6145 & 6.6044 & $82 \ldots \ldots$ & $3 s^{2} 3 p^{3}\left({ }^{2} D\right) 3 d^{2}\left({ }^{3} F\right)$ & ${ }^{2} \mathrm{H}_{9 / 2}^{\circ}$ & 8.1123 & 8.0983 \\
\hline $38 \ldots \ldots$ & $3 \mathrm{~s} 3 \mathrm{p}^{5}\left({ }^{3} \mathrm{P}\right) 3 \mathrm{~d}$ & ${ }^{4} \mathrm{~F}_{3 / 2}^{\circ}$ & 6.6395 & 6.6293 & $83 \ldots \ldots$ & $3 \mathrm{~s}^{2} 3 \mathrm{p}^{3}\left({ }^{2} \mathrm{D}\right) 3 \mathrm{~d}^{2}\left({ }^{1} \mathrm{D}\right)$ & ${ }^{2} \mathrm{~F}_{5 / 2}^{\circ}$ & 8.1176 & 8.1038 \\
\hline $39 \ldots \ldots$ & $3 \mathrm{~s} 3 \mathrm{p}^{5}\left({ }^{3} \mathrm{P}\right) 3 \mathrm{~d}$ & ${ }^{4} D_{7 / 2}^{\circ}$ & 6.8612 & 6.8504 & $84 \ldots \ldots$ & $3 \mathrm{~s}^{2} 3 \mathrm{p}^{3}\left({ }^{2} \mathrm{D}\right) 3 \mathrm{~d}^{2}\left({ }^{1} \mathrm{D}\right)$ & ${ }^{2} \mathrm{D}_{3 / 2}^{\circ}$ & 8.1333 & 8.1211 \\
\hline $40 \ldots \ldots$ & $3 \mathrm{~s} 3 \mathrm{p}^{5}\left({ }^{3} \mathrm{P}\right) 3 \mathrm{~d}$ & ${ }^{4} \mathrm{D}_{1 / 2}^{\circ}$ & 6.8721 & 6.8630 & $\star 85 \ldots \ldots$ & $3 \mathrm{~s}^{2} 3 \mathrm{p}^{3}\left({ }^{2} \mathrm{D}\right) 3 \mathrm{~d}^{2}\left({ }^{3} \mathrm{~F}\right)$ & ${ }^{2} \mathrm{H}_{11 / 2}^{\circ}$ & 8.1336 & 8.1180 \\
\hline $41 \ldots \ldots$ & $3 \mathrm{~s} 3 \mathrm{p}^{5}\left({ }^{3} \mathrm{P}\right) 3 \mathrm{~d}$ & ${ }^{4} \mathrm{D}_{5 / 2}^{\circ}$ & 6.8767 & 6.8662 & $86 \ldots \ldots$ & $3 \mathrm{~s}^{2} 3 \mathrm{p}^{3}\left({ }^{2} \mathrm{P}\right) 3 \mathrm{~d}^{2}\left({ }^{3} \mathrm{~F}\right)$ & ${ }^{4} \mathrm{G}_{5 / 2}^{\circ}$ & 8.1999 & 8.1863 \\
\hline $42 \ldots \ldots$ & $3 \mathrm{~s} 3 \mathrm{p}^{5}\left({ }^{3} \mathrm{P}\right) 3 \mathrm{~d}$ & ${ }^{4} \mathrm{D}_{3 / 2}^{\circ}$ & 6.8777 & 6.8678 & $87 \ldots \ldots$ & $3 s^{2} 3 p^{3}\left({ }^{2} P\right) 3 d^{2}\left({ }^{3} F\right)$ & ${ }^{4} \mathrm{G}_{7 / 2}^{\circ}$ & 8.2122 & 8.1983 \\
\hline $43 \ldots \ldots$ & $3 \mathrm{~s} 3 \mathrm{p}^{5}\left({ }^{3} \mathrm{P}\right) 3 \mathrm{~d}$ & ${ }^{2} \mathrm{~F}_{7 / 2}^{\circ}$ & 6.9369 & 6.9268 & $88 \ldots \ldots$ & $3 s 3 p^{5}\left({ }^{3} P\right) 3 d$ & ${ }^{2} \mathrm{P}_{1 / 2}^{\circ}$ & 8.2169 & 8.2058 \\
\hline $44 \ldots \ldots$ & $3 \mathrm{~s} 3 \mathrm{p}^{5}\left({ }^{3} \mathrm{P}\right) 3 \mathrm{~d}$ & ${ }^{2} \mathrm{~F}_{5 / 2}^{\circ}$ & 6.9965 & 6.9859 & $\star 89 \ldots \ldots$ & $3 s^{2} 3 p^{3}\left({ }^{2} \mathrm{D}\right) 3 d^{2}\left({ }^{3} F\right)$ & ${ }^{2} \mathrm{D}_{5 / 2}^{\circ}$ & 8.2186 & 8.2031 \\
\hline $45 \ldots \ldots$ & $3 \mathrm{~s} 3 \mathrm{p}^{5}\left({ }^{3} \mathrm{P}\right) 3 \mathrm{~d}$ & ${ }^{2} \mathrm{D}_{3 / 2}^{\circ}$ & 7.0612 & 7.0510 & $90 \ldots \ldots$ & $3 \mathrm{~s} 3 \mathrm{p}^{5}\left({ }^{3} \mathrm{P}\right) 3 \mathrm{~d}$ & ${ }^{2} \mathrm{P}_{3 / 2}^{\circ}$ & 8.2235 & 8.2119 \\
\hline
\end{tabular}

\footnotetext{
${ }^{a}$ Present results without Breit and QED corrections.

${ }^{b}$ Present results with Breit and QED corrections.

* The level has changed order with the inclusion of Breit and QED corrections.
} 


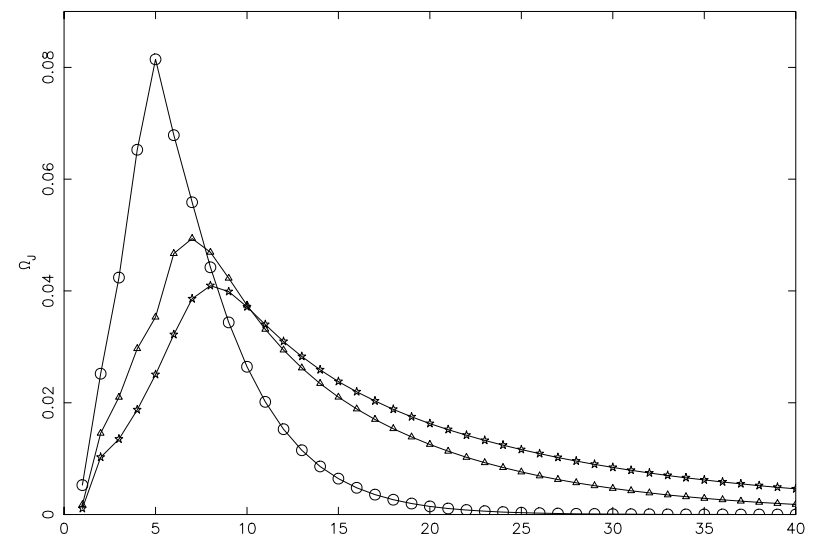

(a)

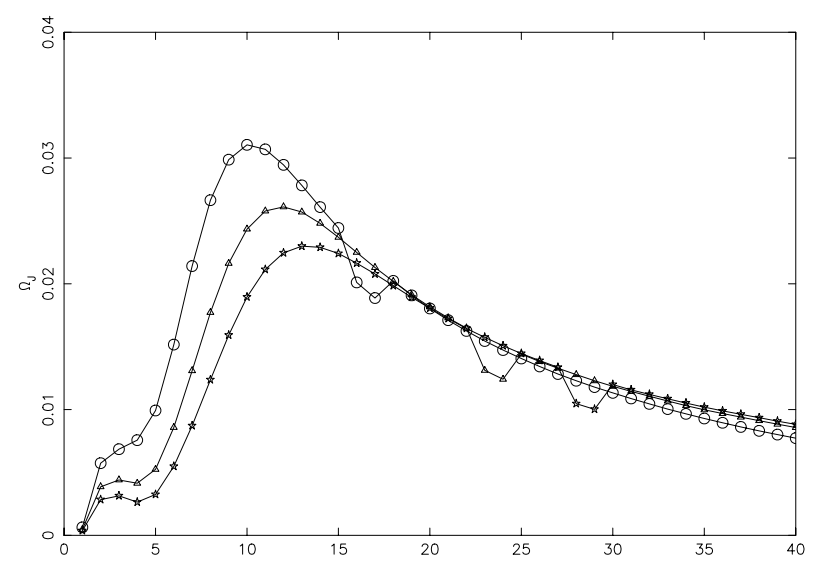

(b)

Fig. 2. Partial collision strengths for the $3 s^{2} 3 p^{5}{ }^{2} P_{3 / 2}^{\circ}-3 s 3 p^{6}{ }^{2} S_{1 / 2}$ (1-3) transition of Fe X, at three energies of: a) 10 Ryd (circles), 30 Ryd (triangles), and 50 Ryd (stars), and b) 100 Ryd (circles), 150 Ryd (triangles), and 200 Ryd (stars).

as $1-3$ shown in Fig. 2 ( $a$ and $b$ ), $\Omega$ have converged within our range of partial waves only at lower energies (below 50 Ryd), and a top-up is required for its convergence at higher energies. Additionally, for some transitions such as $1-3$, the values of $\Omega$ show an unphysical oscillation for two partial waves. This is due to the fact that the long-range potentials have been omitted for these partial waves and only Coulomb functions have been used. This is performed automatically by the program in DSTGF whenever there is a numerical problem in the convergence of the wavefunction in the outer region of the $R$-matrix. The solution to this problem is to increase the number of integration points. In the present calculations, we have restricted this number to 2000, and increasing it further makes the program more computationally demanding. This is not currently feasible keeping in view our resources, and the fact that $\Omega$ have to be computed at a large number of energies. However, as a result of this, $\Omega_{J}$ is up to $\sim 20 \%$ lower than what it should be with the inclusion of long-range potentials. Since it happens for only two partial waves, the net effect on $\sum \Omega_{J}$ is negligible. For example, for the transition shown in Fig. $2 b, \Omega$ values are affected (and are lower) by less than $1 \%$, which is much lower than the accuracy estimates of the calculations.

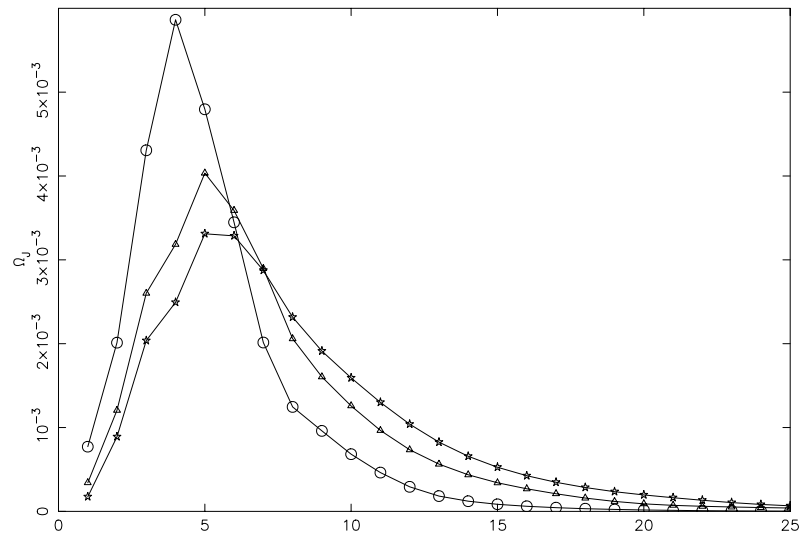

(a)

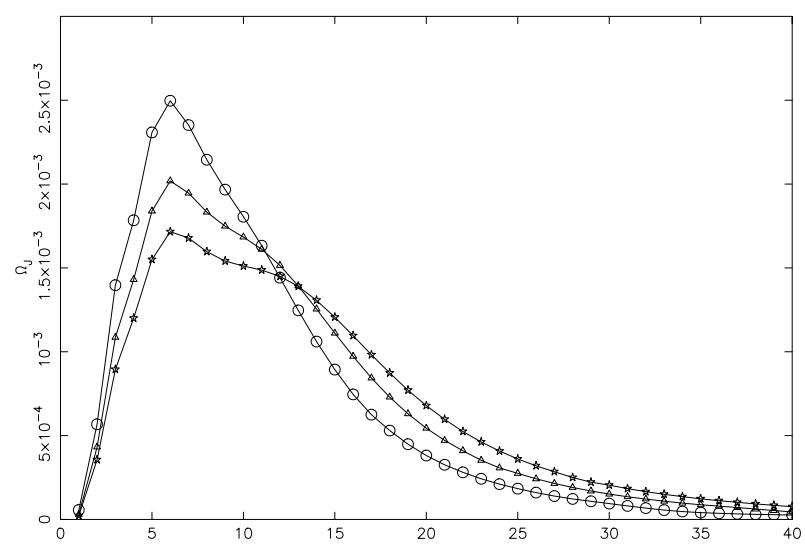

(b)

Fig. 3. Partial collision strengths for the $3 s 3 p^{6}{ }^{2} S_{1 / 2}-3 s^{2} 3 p^{4}\left({ }^{1} S\right) 3 d$ ${ }^{2} \mathrm{D}_{5 / 2}$ (3-26) transition of $\mathrm{Fe} \mathrm{X}$, at three energies of: a) 10 Ryd (circles), 30 Ryd (triangles), and 50 Ryd (stars), and b) 100 Ryd (circles), 150 Ryd (triangles), and 200 Ryd (stars).

In Table 2 we present our results of $\Omega$ for transitions from the lowest 3 levels to higher excited levels, and in a wider energy range up to $200 \mathrm{Ryd}$. The indices adopted to represent a transition are already given in Table 1 . These results of $\Omega$ are not directly applicable in any diagnostic or modelling work, but are very useful in assessing the accuracy of a calculation, and will be helpful for future comparisons. The only significant corresponding data for $\Omega$ available in the literature are of BD, who have reported their results from the DW code, at 5 energies in the range $9.0 \leq E \leq 45.0 \mathrm{Ryd}$. Since their wavefunctions are different from ours (as stated in Sect. 1), disagreements in the values of $\Omega$ are not unexpected. However, for most of the allowed transitions, differences in the two sets of $\Omega$ are in accordance with their corresponding $f$-values, which indicates that there is no unexpected discrepancy. Since the inaccuracy of the BD data has already been highlighted in Sect. 1 (and earlier by PB and SST), our main focus is on comparisons with the other available calculations of PB and SST. Unfortunately, both of these papers have listed values of $\Omega$ at four energies $(18,27$, 36 and 45 Ryd), and only for a few transitions. Nevertheless, in Table 3 we compare all four sets of $\Omega$ (and $f$-values for allowed transitions) for some of the common transitions, which 
Table 3. Comparison of oscillator strengths $(f)$ and collision strengths $(\Omega)$ for some transitions of Fe X. $\left(a \pm b \equiv a \times 10^{ \pm b}\right)$.

\begin{tabular}{|c|c|c|c|c|c|c|c|c|c|c|c|}
\hline \multicolumn{2}{|c|}{ Transition } & \multicolumn{5}{|c|}{ Bhatia \& Doschek (1995) } & \multicolumn{5}{|c|}{ Tayal (2001) } \\
\hline$i$ & $j$ & $f$ & 18.0 & 27.0 & 36.0 & 45.0 & $f$ & 18.0 & 27.0 & 36.0 & 45.0 Ryd \\
\hline 1 & 2 & & $2.358-1$ & $2.251-1$ & $2.188-1$ & $2.143-1$ & & 0.368 & 0.345 & 0.328 & 0.318 \\
\hline 1 & 3 & 0.0179 & $4.212-1$ & $4.414-1$ & $4.610-1$ & $4.791-1$ & 0.028 & 0.607 & 0.669 & 0.714 & 0.754 \\
\hline 1 & 4 & $1.78-5$ & $3.915-2$ & $2.552-2$ & $1.787-2$ & $1.325-2$ & & 0.044 & 0.029 & 0.020 & 0.015 \\
\hline 1 & 5 & & $5.929-2$ & $3.834-2$ & $2.651-2$ & $1.933-2$ & & 0.066 & 0.043 & 0.030 & 0.022 \\
\hline 2 & 3 & 0.0183 & $2.255-1$ & $2.394-1$ & $2.515-1$ & $2.622-1$ & 0.028 & 0.327 & 0.360 & 0.383 & 0.404 \\
\hline 2 & 4 & & $1.200-2$ & $7.893-3$ & $5.582-3$ & $4.179-3$ & & 0.013 & 0.009 & 0.006 & 0.005 \\
\hline 2 & 5 & & 7.918-3 & $5.047-3$ & $3.470-3$ & $2.524-3$ & & 0.009 & 0.006 & 0.004 & 0.003 \\
\hline 3 & 4 & & $1.999-3$ & $1.3-1-3$ & $9.220-4$ & $6.940-4$ & & & & & \\
\hline 3 & 5 & & $2.621-3$ & $1.672-3$ & $1.158-3$ & $8.490-4$ & & & & & \\
\hline
\end{tabular}

\begin{tabular}{cllllllllllll}
\hline \multicolumn{1}{c}{ Transition } & \multicolumn{4}{c}{ Pelan \& Berrington (2001) } & \multicolumn{5}{c}{ Present results } \\
\hline$i$ & $j$ & $f$ & 18.0 & 27.0 & 36.0 & 45.0 & $f$ & 18.0 & 27.0 & 36.0 & 45.0 Ryd \\
\hline 1 & 2 & & & & & & & 0.2111 & 0.2057 & 0.2031 & 0.2018 \\
1 & 3 & 0.0257 & 0.356 & 0.363 & 0.361 & 0.361 & 0.0273 & 0.5961 & 0.6520 & 0.6952 & 0.7300 \\
1 & 4 & $5.20-5$ & 0.044 & 0.028 & 0.020 & 0.015 & $1.00-4$ & $4.55-2$ & $3.00-2$ & $2.13-2$ & $1.61-2$ \\
1 & 5 & & 0.0662 & 0.0423 & 0.0293 & 0.0213 & & $6.71-2$ & $4.31-2$ & $2.97-2$ & $2.16-2$ \\
2 & 3 & 0.0257 & 0.186 & 0.188 & 0.188 & 0.188 & 0.0267 & 0.3164 & 0.3464 & 0.3692 & 0.3875 \\
2 & 4 & & 0.0140 & 0.0085 & 0.0060 & 0.0045 & & $1.34-2$ & $8.79-3$ & $6.20-3$ & $4.64-3$ \\
2 & 5 & & & & & & & $9.05-3$ & $5.75-3$ & $3.94-3$ & $2.85-3$ \\
3 & 4 & & 0.0020 & 0.0013 & 0.0010 & 0.0008 & & $2.09-3$ & $1.41-3$ & $1.03-3$ & $8.00-4$ \\
3 & 5 & & & & & & & $2.69-3$ & $1.74-3$ & $1.22-3$ & $9.03-4$ \\
\hline
\end{tabular}

will serve our purpose of making an assessment of the accuracy of the calculations.

As stated earlier, for allowed transitions (such as 1-3 and 2-3) the $\Omega$ values of BD differ with those of ours or of SST by $\sim 50 \%$ over the entire energy range. However, these differences are clearly understandable because the corresponding $f$-values also differ by the same amount. Among the forbidden transitions, noticeable differences are for the 1-2 transition, for which ours and the BD values of $\Omega$ are comparable, but those of SST are higher by $\sim 50 \%$. For this transition, PB have not reported their results, but in general their values of $\Omega$ for forbidden transitions are in agreement with all other calculations. However, their results for the allowed transitions are in complete contrast with other work. In spite of their $f$-values for the 1-3 and 2-3 transitions being comparable with those of ours or of SST, the PB values of $\Omega$ are lower by a factor of two, and are not in accordance with the expected behaviour. Similarly, in spite of their $f$-values being higher than those of BD, their values of $\Omega$ are the lowest. In the absence of much detail about their calculations, it is not straightforward to identify the exact source of this obvious discrepancy in the PB data for allowed transitions. However, based on our experience on a number of Fe ions, and similar discrepancies observed earlier among different calculations for some of these ions, such as Fe XI (Aggarwal \& Keenan 2003a,b), Fe XIII (Aggarwal \& Keenan 2004a, 2005), and Fe XV (Aggarwal et al. 2000, 2001, 2003a), we make an attempt to understand this discrepancy.
Since the $\Omega$ values of PB are (nearly) constant for the $1-3$ and 2-3 allowed transitions, in the entire energy range of 18-45 Ryd, it gives us an impression that for at least these energies, their results are not converged. Since they claim to have included all partial waves with angular momentum $J \leq$ 56 , the results for $\Omega$ must have converged, as is apparent from Fig. 2a for the 1-3 transition. Additionally, values of $\Omega$ for allowed transitions generally increase with increasing energy, and this is also the trend confirmed by the other three calculations of BD, SST and ours. To elaborate further, in Fig. 4 we show the variation of $\Omega$ with the increasing addition of partial waves, for the 1-3 transition at three energies of 18,27 and 45 Ryd. It is clear from this figure that $\Omega$ have (nearly) converged in the partial waves range with $J \leq 39$, at all three energies. It also clearly shows the cross-over from the contributions of lower partial waves to the higher ones. Up to a certain range (below $J=20$ in the present case) the lower partial waves dominate, which means that if only $J \leq 20$ are included, $\sum \Omega_{J}$ will decrease with increasing energy. However, as the contribution of higher partial waves is included, the reverse becomes true, i.e. $\sum \Omega_{J}$ becomes higher at higher energies. In the past, due to a scarcity of computational resources, it was a general practice to gradually increase the contribution of partial waves with increasing electron energy. Therefore, we suspect that PB might have included all partial waves with $J \leq 56$ for energies towards the higher end of their calculations of $\Omega$, but a limited range of partial waves was probably included for the 


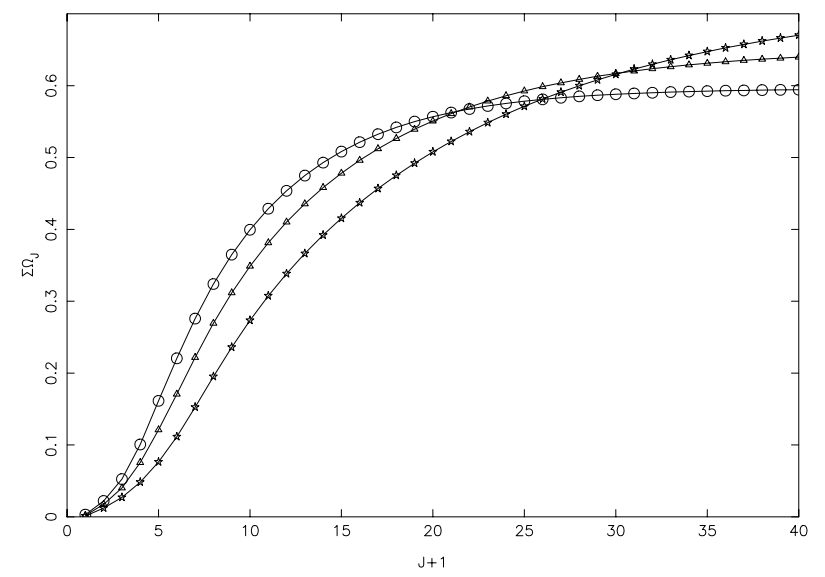

Fig. 4. Partial sums of collision strengths for the $3 \mathrm{~s}^{2} 3 \mathrm{p}^{5}$ ${ }^{2} \mathrm{P}_{3 / 2}^{\circ}-3 \mathrm{~s} 3 \mathrm{p}^{6}{ }^{2} \mathrm{~S}_{1 / 2}$ (1-3) transition of $\mathrm{Fe} \mathrm{X}$, at three energies of 18 Ryd (circles), 27 Ryd (triangles), and 45 Ryd (stars).

calculations of $\Omega$ at energies below $\sim 50$ Ryd. A detailed comparison of our calculations, for both 1-3 and 2-3 transitions, with their results listed in Table 3, indicates that they probably included $J \leq 8, J \leq 10$ and $J \leq 12$ for calculating values of $\Omega$ at $E=18,27$ and 45 Ryd, respectively. Furthermore, apart from what is shown in Figs. 2a and 4, our above analysis is based on exactly the same discrepancy observed in the Breit-Pauli calculations of Eissner et al. (1999) for allowed transitions in Fe XV - see particularly Fig. 5 of Aggarwal et al. (2000) and note the step-wise increase in the $\Omega$ values of Eissner et al., and Figs. 1-4 of Aggarwal et al. (2001). Furthermore, in a recent paper on Fe XXIII, Chidichimo et al. (2005) have also noted the non-inclusion of higher partial waves at lower energies in their earlier work (Chidichimo et al. 1999).

To conclude, we can state with confidence that for allowed transitions, and at least at energies below $\sim 50 \mathrm{Ryd}$, the $\Omega$ values of Pelan \& Berrington (2001) are not converged, due to the inclusion of a limited range of partial waves. Since this energy region is very important for the calculations of $\Upsilon$ at temperatures below $7 \times 10^{6} \mathrm{~K}\left(\equiv \log T_{\mathrm{e}} \leq 6.85 \mathrm{~K}\right)$, the reported results of $\mathrm{PB}$ for the effective collision strengths are expected to be underestimated, especially for the allowed transitions, in the entire temperature range of their calculations. Some of the forbidden transitions will also be affected (see Figs. 1a and 3a for the 1-2 and 3-26), but not to the same extent as the allowed ones. We discuss this further in Sect. 4.

Finally, in Table 4 we demonstrate the effect of top-up for two forbidden (namely, 1-2 and 5-16) and two allowed (namely, 1-28 and 2-3) transitions, at three energies of 100, 150 and 200 Ryd. It is clear from this table that partial waves with $J \leq 9$ are inadequate for accurate determination of $\Omega$ for both types of transitions. Similarly, partial waves with $J \leq 19$ may result up to $20 \%$ underestimation in the values of $\Omega$, especially for the allowed transitions. However, further addition of 10 more partial waves provides results with comparable accuracy as achieved within our range of calculations with $J \leq 39$. Therefore, based on this comparison and the behaviour of partial $\Omega$ s shown earlier in Figs. 1-4, we can state with confidence that the accuracy of our $\Omega$ values is better than $10 \%$, at all energies and for all transitions.

\section{Effective collision strengths}

Effective collision strengths $\Upsilon$ are obtained after integrating $\Omega$ over a Maxwellian distribution of electron velocities, i.e.

$\Upsilon\left(T_{\mathrm{e}}\right)=\int_{0}^{\infty} \Omega(E) \exp \left(-E_{j} / k T_{\mathrm{e}}\right) \mathrm{d}\left(E_{j} / k T_{\mathrm{e}}\right)$

where $E_{j}$ is the incident energy of the electron with respect to the final state of the transition, $k$ is Boltzmann's constant, and $T_{\mathrm{e}}$ is the electron temperature in $\mathrm{K}$. Once the value of $\Upsilon$ is known for a transition, the corresponding value of the excitation $q(i, j)$ and de-excitation $q(j, i)$ rate coefficients can be easily obtained from the following simple relations:

$q(i, j)=\frac{8.63 \times 10^{-6}}{\omega_{i} T_{\mathrm{e}}^{1 / 2}} \Upsilon \exp \left(-E_{i j} / k T_{\mathrm{e}}\right) \quad \mathrm{cm}^{3} \mathrm{~s}^{-1}$

and

$q(j, i)=\frac{8.63 \times 10^{-6}}{\omega_{j} T_{\mathrm{e}}^{1 / 2}} \Upsilon \mathrm{cm}^{3} \mathrm{~s}^{-1}$,

where $\omega_{i}$ and $\omega_{j}$ are the statistical weights of the initial $(i)$ and final $(j)$ states, respectively, and $E_{i j}$ is the transition energy.

Since the threshold energy region is dominated by numerous resonances, $\Omega$ must be computed at a large number of energies in order to delineate these resonances. We have performed our calculations of $\Omega$ at over 5400 energies in the threshold region. Close to thresholds $(\sim 0.1$ Ryd above a threshold) the energy mesh is $0.001 \mathrm{Ryd}$, and away from thresholds is 0.002 Ryd. Thus care has been taken to include as many resonances as possible, and with as fine a resolution as is computationally feasible. The values of $\Upsilon$ so computed are listed in Table 5 at a series of electron temperatures in the range $5.0 \leq \log T_{\mathrm{e}} \leq 7.0 \mathrm{~K}$, which is fully sufficient for the application of the data to solar, astrophysical and fusion plasmas.

Before we compare our $\Upsilon$ values, we would like to mention that the energy range of our calculations (i.e. $210 \mathrm{Ryd}$ ) is fully sufficient to determine results up to $T_{\mathrm{e}}=10^{7} \mathrm{~K}$, which corresponds to $\sim 63$ Ryd in energy units. Hence, our included energy range is more than three times the highest temperature considered in the calculations. To assess the effect of various energy ranges, we have performed an exercise at a temperature of $10^{6} \mathrm{~K}$, including energies up to $15,21,27,33$ and $40 \mathrm{Ryd}$, which are up to 5 times this temperature of $\sim 6.3 \mathrm{Ryd}$, including the highest threshold energy of $\sim 8$ Ryd. Results for resonance transitions obtained with up to one and two times the $T_{\mathrm{e}}$ energies differ up to $35 \%$, whereas the corresponding differences with up to two and three times the $T_{\mathrm{e}}$ energies are within $11 \%$. Extension of the energy range up to four times yields the $\Upsilon$ values within $4 \%$, which further converge within $2 \%$ with the inclusion of higher energies up to five times the $T_{\mathrm{e}}$. Therefore, we see no need to extrapolate our values of $\Omega$ to determine results for $\Upsilon$.

In Table 6 we compare our results of $\Upsilon$ with those of SST and PB for common transitions, from the lowest three levels to higher excited levels, at three common temperatures 
Table 4. Convergence of collision strengths for some transitions of Fe X. $a \pm b \equiv a \times 10^{ \pm b}$.

(a) Forbidden transitions.

\begin{tabular}{|c|c|c|c|c|c|c|}
\hline \multirow{2}{*}{$\begin{array}{l}\text { Transition } \\
\text { Partial waves }\end{array}$} & \multicolumn{3}{|c|}{$1-2$} & \multicolumn{3}{|c|}{$5-16$} \\
\hline & 100 & 150 & 200 & 100 & 150 & 200 Ryd \\
\hline$J \leq 9$ & $1.20-1$ & $9.87-2$ & $8.43-2$ & $1.20-1$ & $9.92-2$ & $8.46-2$ \\
\hline+ top-up & $2.21-1$ & $4.76-1$ & $8.74-1$ & $2.04-1$ & $2.43-1$ & $3.22-1$ \\
\hline$J \leq 19$ & $1.82-1$ & $1.72-1$ & $1.62-1$ & $1.83-1$ & $1.74-1$ & $1.64-1$ \\
\hline+ top-up & $1.90-1$ & $1.85-1$ & $1.81-1$ & $1.97-1$ & $1.98-1$ & $1.98-1$ \\
\hline$J \leq 29$ & $1.95-1$ & $1.91-1$ & $1.87-1$ & $1.96-1$ & $1.93-1$ & $1.89-1$ \\
\hline+ top-up & $1.98-1$ & $1.96-1$ & $1.96-1$ & $2.02-1$ & $2.02-1$ & $2.07-1$ \\
\hline$J \leq 39$ & $1.99-1$ & $1.98-1$ & $1.96-1$ & $2.00-1$ & $1.99-1$ & $1.97-1$ \\
\hline+ top-up & $2.01-1$ & $2.00-1$ & $1.99-1$ & $2.03-1$ & $2.04-1$ & $2.04-1$ \\
\hline
\end{tabular}

(b) Allowed transitions.

\begin{tabular}{lllllll}
\hline Transition & \multicolumn{3}{c}{$1-28$} & & \multicolumn{3}{c}{$2-3$} \\
\hline Partial waves & 100 & 150 & 200 & 100 & 150 & 200 Ryd \\
\hline$J \leq 9$ & $1.83+0$ & $1.23-0$ & $8.78-1$ & $7.43-2$ & $4.92-2$ & $3.48-2$ \\
+ top-up & $6.49+0$ & $6.35+0$ & $6.32+0$ & $2.97-1$ & $2.90-1$ & $2.88-1$ \\
$J \leq 19$ & $4.80+0$ & $4.13+0$ & $3.55+0$ & $1.98-1$ & $1.68-1$ & $1.45-1$ \\
+ top-up & $9.45+0$ & $9.25+0$ & $8.99+0$ & $4.20-1$ & $4.09-1$ & $3.98-1$ \\
$J \leq 29$ & $6.30+0$ & $5.82+0$ & $5.32+0$ & $2.71-1$ & $2.40-1$ & $2.17-1$ \\
+ top-up & $1.00+1$ & $1.07+1$ & $1.08+1$ & $4.46-1$ & $4.68-1$ & $4.71-1$ \\
$J \leq 39$ & $7.09+0$ & $6.89+0$ & $6.53+0$ & $3.21-1$ & $2.92-1$ & $2.70-1$ \\
+ top-up & $1.00+1$ & $1.07+1$ & $1.12+0$ & $4.46-1$ & $4.68-1$ & $4.88-1$ \\
\hline
\end{tabular}

of $\log T_{\mathrm{e}}=5.4,6.0$ and $6.6 \mathrm{~K}$. Although all three sets of calculations are based on the $R$-matrix method, included CI and relativistic effects in the generation of wavefunctions, and have resolved resonances in thershold region, the values of $\Upsilon$ show poor agreement for a majority of transitions. Different sets of results differ by up to a factor of two. For example, the $\Upsilon$ values of SST and PB differ for the 1-4 and 2-4 transitions, SST and ours for the 1-5 and 1-9, and PB and ours for the 1-3 and 1-5 transitions. However, such large differences among different calculations are fully understandable, but before we explain this we would like to first mention the specific differences between our calculations and those of SST and PB. Firstly, our calculations are fully relativistic and in $j j$ coupling, which not only properly accounts for the relativistic effects, but also shows comparatively more resonances because of the inclusion of fine-structure in the definition of channel coupling. In contrast, the calculations of SST and PB are semi-relativistic in the $L S J$ coupling scheme, and do not account for the two-body relativistic operators. Secondly, for the generation of wavefunctions our calculations include larger CI than those of SST or PB, which improves the accuracy of the energy levels and radiative rates, and subsequently of $\Omega$ and $\Upsilon$. Thirdly, our calculations include a larger range of partial waves $(J \leq 39)$ than those of $\operatorname{SST}(J \leq 20)$ or of PB (see Sect. 3). This improves the accuracy of the $\Omega$ values, not only for the allowed transitions but for the forbidden ones also, as already discussed in Sect. 3. Similarly, our calculations cover a wider range of energy, i.e. $E \leq 210$ Ryd in comparison to $E \leq 90$ Ryd of SST. PB have included a higher energy range, but to no additional advantage, because to calculate values of $\Upsilon$ up to $T_{\mathrm{e}}=10^{7} \mathrm{~K}, E \leq 210$ Ryd is more than sufficient, as discussed above. Finally, our calculations include 90 levels, whereas those of SST and PB include 54 and 31 levels, respectively. The energy mesh of our calculations and those of PB are comparable ( $\Delta E \leq 0.002 \mathrm{Ryd}$ ), whereas that of SST is comparatively coarse, i.e. $\Delta E \leq 0.005$ Ryd. In summary, the calculations of PB or ours are comparatively more sophisticated than those of SST, yet there is a better agreement of our $\Upsilon$ results with those of SST than of PB. We explain this below.

In Figs. 5-7 we show our values of $\Omega$ in the threshold region for the $1-2,1-3$ and 2-3 transitions, respectively. SST has shown his results for these transitions in his Figs. 1-3 (Tayal 2000), and PB have shown their results for the 1-3 and 2-3 transitions in their Fig. 2. A comparison of these figures clearly shows that resonances in our calculations are denser, because of the reasons explained above. Therefore, it is no surprise that our results of $\Upsilon$ are higher than those of SST, especially for the forbidden transitions, such as 1-2, $2-4$ and $3-5$. For some of the allowed transitions (such as 1-9, 1-12 and 2-28), part of the differences between our results and his are probably because of the similar differences in the corresponding $f$-values, although it cannot be verified because he 
Table 6. Comparison of effective collision strengths ( $\Upsilon$ ) for some transitions of Fe X. $\left(a \pm b \equiv a \times 10^{ \pm b}\right.$.)

\begin{tabular}{|c|c|c|c|c|c|c|c|c|c|c|}
\hline \multicolumn{2}{|c|}{ Transition } & \multicolumn{3}{|c|}{ Tayal (2001) } & \multirow{2}{*}{\multicolumn{3}{|c|}{$\begin{array}{c}\text { Pelan \& Berrington }(2001) \\
\text { Temperature }\left(\log T_{2}, \mathrm{~K}\right)\end{array}$}} & \multicolumn{3}{|c|}{ Present results } \\
\hline \multirow[b]{2}{*}{$i$} & \multirow[b]{2}{*}{$j$} & \multicolumn{3}{|c|}{ Temperature $\left(\log T_{\mathrm{e}}, \mathrm{K}\right)$} & & & & \multicolumn{3}{|c|}{ Temperature $\left(\log T_{\mathrm{e}}, \mathrm{K}\right)$} \\
\hline & & 5.40 & 6.00 & 6.60 & 5.40 & 6.00 & 6.60 & 5.40 & 6.00 & 6.60 \\
\hline 1 & 2 & 2.232 & 1.130 & 0.494 & $2.970-0$ & $1.790-0$ & $7.300-1$ & $3.118-0$ & $1.813-0$ & $7.392-1$ \\
\hline 1 & 3 & 0.753 & 0.647 & 0.667 & $7.939-1$ & $5.133-1$ & $4.008-1$ & $1.202-0$ & $8.902-1$ & 7.444-1 \\
\hline 1 & 4 & 0.368 & 0.162 & 0.067 & $1.742-1$ & $1.045-1$ & $5.222-2$ & $5.863-1$ & $2.293-1$ & $8.620-2$ \\
\hline 1 & 5 & 0.475 & 0.218 & 0.093 & $2.389-1$ & $1.468-1$ & $7.492-2$ & $9.143-1$ & $3.424-1$ & $1.263-1$ \\
\hline 1 & 6 & 0.234 & 0.100 & 0.040 & $9.933-2$ & $5.968-2$ & $2.979-2$ & $2.943-1$ & $1.148-1$ & $4.448-2$ \\
\hline 1 & 7 & 0.103 & 0.044 & 0.017 & $4.176-2$ & $2.500-2$ & $1.215-2$ & $1.967-1$ & $7.060-2$ & $2.430-2$ \\
\hline 1 & 9 & 0.143 & 0.064 & 0.026 & $1.702-1$ & $1.036-1$ & $5.142-2$ & $4.370-1$ & $2.224-1$ & $8.621-2$ \\
\hline 1 & 8 & 0.273 & 0.129 & 0.053 & $7.151-2$ & $4.285-2$ & $2.321-2$ & $1.505-1$ & $7.076-2$ & $2.873-2$ \\
\hline 1 & 10 & 0.219 & 0.098 & 0.039 & $1.281-1$ & $7.439-2$ & $3.679-2$ & $2.176-1$ & $1.071-1$ & $4.622-2$ \\
\hline 1 & 11 & 0.156 & 0.071 & 0.031 & $8.460-2$ & $4.880-2$ & $2.392-2$ & $1.343-1$ & $6.965-2$ & $3.282-2$ \\
\hline 1 & 12 & 0.118 & 0.055 & 0.025 & $1.132-1$ & $7.286-2$ & $4.108-2$ & $2.316-1$ & $1.242-1$ & $5.329-2$ \\
\hline 1 & 13 & 0.253 & 0.124 & 0.066 & $6.973-2$ & $4.195-2$ & $1.989-2$ & $3.655-1$ & $1.997-1$ & $8.948-2$ \\
\hline 1 & 14 & 0.213 & 0.117 & 0.075 & $7.923-2$ & $5.739-2$ & $2.952-2$ & $3.221-1$ & $1.802-1$ & $9.809-2$ \\
\hline 1 & 15 & 0.070 & 0.036 & 0.023 & $9.438-2$ & $5.733-2$ & $3.106-2$ & $6.069-2$ & $3.666-2$ & $2.312-2$ \\
\hline 1 & 16 & 0.131 & 0.058 & 0.024 & $5.434-2$ & $3.293-2$ & $2.400-2$ & $1.338-1$ & $6.642-2$ & $2.565-2$ \\
\hline 1 & 17 & 0.230 & 0.117 & 0.062 & $1.343-1$ & $9.302-2$ & $6.585-2$ & $6.413-1$ & $3.457-1$ & $1.418-1$ \\
\hline 1 & 18 & 0.387 & 0.167 & 0.081 & $1.351-1$ & $9.067-2$ & $6.485-2$ & $6.437-1$ & $2.965-1$ & $1.316-1$ \\
\hline 1 & 19 & 0.267 & 0.135 & 0.118 & $1.619-1$ & $9.527-2$ & $6.761-2$ & $3.754-1$ & $1.914-1$ & $7.929-2$ \\
\hline 1 & 20 & & & & $1.627-1$ & $1.100-1$ & $8.230-2$ & $5.610-1$ & $2.523-1$ & $1.218-1$ \\
\hline 1 & 21 & & & & $1.177-1$ & $6.502-2$ & $2.889-2$ & $2.794-1$ & $1.210-1$ & $4.433-2$ \\
\hline 1 & 22 & 0.197 & 0.088 & 0.044 & $1.101-1$ & $6.217-2$ & $3.300-2$ & $3.002-1$ & $1.321-1$ & $5.597-2$ \\
\hline 1 & 23 & 0.236 & 0.102 & 0.057 & $1.045-1$ & $6.480-2$ & $4.470-2$ & $1.804-1$ & $9.901-2$ & $6.153-2$ \\
\hline 1 & 24 & 0.288 & 0.126 & 0.073 & $1.288-1$ & $8.221-2$ & $6.221-2$ & $2.194-1$ & $1.040-1$ & $6.415-2$ \\
\hline 1 & 25 & 0.152 & 0.080 & 0.063 & $5.803-2$ & $4.534-2$ & $3.937-2$ & $2.045-1$ & $9.789-2$ & $6.087-2$ \\
\hline 1 & 26 & 0.217 & 0.092 & 0.054 & $5.941-2$ & $4.350-2$ & $4.242-2$ & $1.326-1$ & $6.780-2$ & $4.115-2$ \\
\hline 1 & 27 & 1.709 & 2.003 & 2.763 & $1.908-0$ & $1.933-0$ & $1.950-0$ & $1.887-0$ & $2.080-0$ & $2.649-0$ \\
\hline 1 & 28 & 3.360 & 3.847 & 5.286 & $3.856-0$ & $3.934-0$ & $4.018-0$ & $4.081-0$ & $4.224-0$ & $5.337-0$ \\
\hline 1 & 29 & 0.494 & 0.511 & 0.681 & $4.783-1$ & $4.924-1$ & $5.608-1$ & $5.618-1$ & $5.518-1$ & $6.788-1$ \\
\hline 1 & 30 & 5.628 & 6.650 & 9.217 & $6.805-0$ & $7.032-0$ & $7.171-0$ & $6.476-0$ & $7.259-0$ & $9.355-0$ \\
\hline 1 & 31 & 0.322 & 0.292 & 0.363 & $2.161-1$ & $2.274-1$ & $2.690-1$ & $1.909-1$ & $1.987-1$ & $2.406-1$ \\
\hline 2 & 3 & 0.380 & 0.340 & 0.356 & $3.508-1$ & $2.481-1$ & $2.039-1$ & $5.769-1$ & $4.661-1$ & $3.953-1$ \\
\hline 2 & 4 & 0.164 & 0.066 & 0.025 & $5.955-2$ & $3.416-2$ & $1.662-2$ & $4.003-1$ & $1.305-1$ & $4.159-2$ \\
\hline 2 & 5 & 0.160 & 0.062 & 0.022 & $5.393-2$ & $2.842-2$ & $1.270-2$ & $1.808-1$ & $6.708-2$ & $2.292-2$ \\
\hline 2 & 6 & 0.125 & 0.054 & 0.022 & $5.324-2$ & $3.237-2$ & $1.627-2$ & $1.319-1$ & $5.434-2$ & $2.181-2$ \\
\hline 2 & 7 & 0.067 & 0.031 & 0.013 & $3.206-2$ & $2.058-2$ & $1.077-2$ & $8.254-2$ & $3.457-2$ & $1.445-2$ \\
\hline 2 & 8 & 0.087 & 0.039 & 0.016 & $2.919-2$ & $1.508-2$ & $6.590-3$ & $4.530-2$ & $1.960-2$ & $7.596-3$ \\
\hline 2 & 9 & 0.074 & 0.036 & 0.021 & $3.117-2$ & $2.159-2$ & $1.185-2$ & $6.843-2$ & $3.856-2$ & $2.489-2$ \\
\hline 2 & 10 & 0.102 & 0.048 & 0.020 & $4.983-2$ & $2.856-2$ & $1.362-2$ & $7.923-2$ & $3.996-2$ & $1.648-2$ \\
\hline 2 & 11 & 0.084 & 0.041 & 0.017 & $5.393-2$ & $3.136-2$ & $1.508-2$ & $7.515-2$ & $3.934-2$ & $1.724-2$ \\
\hline 2 & 12 & 0.059 & 0.028 & 0.011 & $7.865-2$ & $4.837-2$ & $2.264-2$ & $1.955-1$ & $9.893-2$ & $4.189-2$ \\
\hline 2 & 13 & 0.140 & 0.070 & 0.035 & $4.683-2$ & $2.706-2$ & $1.835-2$ & $2.194-1$ & $1.108-1$ & $4.292-2$ \\
\hline 2 & 14 & 0.099 & 0.049 & 0.031 & $3.081-2$ & $1.936-2$ & $9.344-3$ & $2.018-1$ & $9.542-2$ & $3.901-2$ \\
\hline 2 & 15 & 0.035 & 0.018 & 0.010 & $3.601-2$ & $2.157-2$ & $1.060-2$ & $2.118-2$ & $1.356-2$ & $8.386-3$ \\
\hline 2 & 16 & 0.090 & 0.045 & 0.020 & $5.164-2$ & $3.272-2$ & $2.405-2$ & $1.598-1$ & $7.657-2$ & $2.840-2$ \\
\hline 2 & 17 & 0.081 & 0.035 & 0.015 & $4.712-2$ & $2.662-2$ & $1.277-2$ & $1.053-1$ & $5.557-2$ & $2.432-2$ \\
\hline 2 & 18 & 0.118 & 0.053 & 0.027 & $8.664-2$ & $4.518-2$ & $2.053-2$ & $1.398-1$ & $5.981-2$ & $2.052-2$ \\
\hline 2 & 19 & 0.171 & 0.093 & 0.050 & $7.566-2$ & $5.034-2$ & $3.169-2$ & $1.537-1$ & $8.785-2$ & $4.327-2$ \\
\hline 2 & 20 & & & & $9.240-2$ & $5.633-2$ & $2.808-2$ & $3.740-1$ & $1.291-1$ & $4.635-2$ \\
\hline 2 & 21 & & & & $1.118-1$ & $7.448-2$ & $5.606-2$ & $1.966-1$ & $1.058-1$ & $6.798-2$ \\
\hline
\end{tabular}


Table 6. continued.

\begin{tabular}{|c|c|c|c|c|c|c|c|c|c|c|}
\hline \multicolumn{2}{|c|}{ Transition } & \multicolumn{3}{|c|}{ Tayal (2001) } & \multicolumn{3}{|c|}{ Pelan \& Berrington (2001) } & \multicolumn{3}{|c|}{ Present results } \\
\hline \multirow[b]{2}{*}{$i$} & \multirow[b]{2}{*}{$j$} & \multicolumn{3}{|c|}{ Temperature $\left(\log T_{\mathrm{e}}, \mathrm{K}\right)$} & \multicolumn{3}{|c|}{ Temperature $\left(\log T_{\mathrm{e}}, \mathrm{K}\right)$} & \multicolumn{3}{|c|}{ Temperature $\left(\log T_{\mathrm{e}}, \mathrm{K}\right)$} \\
\hline & & 5.40 & 6.00 & 6.60 & 5.40 & 6.00 & 6.60 & 5.40 & 6.00 & 6.60 \\
\hline 2 & 22 & 0.152 & 0.066 & 0.032 & $8.446-2$ & $5.010-2$ & $2.847-2$ & $2.931-1$ & $1.551-1$ & $6.390-2$ \\
\hline 2 & 23 & 0.122 & 0.053 & 0.033 & $6.416-2$ & $3.916-2$ & $3.245-2$ & $1.223-1$ & $6.197-2$ & $3.494-2$ \\
\hline 2 & 24 & 0.125 & 0.054 & 0.029 & $6.110-2$ & $4.136-2$ & $3.164-2$ & $7.865-2$ & $4.544-2$ & $2.991-2$ \\
\hline 2 & 25 & 0.093 & 0.036 & 0.016 & $3.808-2$ & $2.613-2$ & $1.251-2$ & $3.966-2$ & $2.529-2$ & $1.879-2$ \\
\hline 2 & 26 & 0.122 & 0.054 & 0.028 & $4.474-2$ & $3.155-2$ & $2.069-2$ & $1.486-1$ & $6.576-2$ & $3.106-2$ \\
\hline 2 & 27 & 0.624 & 0.715 & 0.958 & $6.641-1$ & $6.714-1$ & $7.446-1$ & $6.783-1$ & $7.521-1$ & $9.556-1$ \\
\hline 2 & 28 & 0.274 & 0.224 & 0.263 & $1.400-1$ & $1.393-1$ & $1.828-1$ & $1.552-1$ & $1.291-1$ & $1.438-1$ \\
\hline 2 & 29 & 1.337 & 1.550 & 2.136 & $1.520-0$ & $1.548-0$ & $1.549-0$ & $1.418-0$ & $1.610-0$ & $2.080-0$ \\
\hline 2 & 30 & 0.130 & 0.053 & 0.024 & $2.923-2$ & $2.191-2$ & $1.624-2$ & $6.315-2$ & $3.047-2$ & $1.874-2$ \\
\hline 2 & 31 & 3.545 & 4.185 & 5.811 & $4.289-0$ & $4.431-0$ & $4.500-0$ & $4.056-0$ & $4.625-0$ & $5.977-0$ \\
\hline 3 & 4 & 0.082 & 0.031 & 0.010 & $7.320-2$ & $3.511-2$ & $1.180-2$ & $1.352-1$ & $5.156-2$ & $1.576-2$ \\
\hline 3 & 5 & 0.095 & 0.036 & 0.011 & $7.490-2$ & $3.613-2$ & $1.232-2$ & $1.206-1$ & $4.800-2$ & $1.508-2$ \\
\hline 3 & 6 & 0.062 & 0.023 & 0.007 & $5.718-2$ & $2.846-2$ & $9.616-3$ & $1.059-1$ & $3.802-2$ & $1.137-2$ \\
\hline 3 & 7 & 0.032 & 0.013 & 0.004 & $3.368-2$ & $1.877-2$ & $6.472-3$ & $5.473-2$ & $2.008-2$ & $5.993-3$ \\
\hline 3 & 8 & 0.024 & 0.008 & 0.002 & $4.523-2$ & $1.926-2$ & $5.966-3$ & $4.152-2$ & $1.754-2$ & $5.298-3$ \\
\hline 3 & 9 & 0.076 & 0.031 & 0.009 & $1.418-1$ & $7.953-2$ & $2.662-2$ & $6.101-2$ & $2.742-2$ & $8.571-3$ \\
\hline 3 & 10 & 0.020 & 0.008 & 0.002 & $4.355-2$ & $1.893-2$ & $5.838-3$ & $4.211-2$ & $1.767-2$ & $5.250-3$ \\
\hline 3 & 11 & 0.016 & 0.007 & 0.003 & $3.996-2$ & $1.757-2$ & $5.655-3$ & $3.980-2$ & $1.753-2$ & $5.573-3$ \\
\hline 3 & 12 & 0.020 & 0.010 & 0.006 & $1.864-1$ & $1.074-1$ & $3.684-2$ & $6.341-2$ & $3.089-2$ & $1.059-2$ \\
\hline 3 & 13 & 0.119 & 0.050 & 0.015 & $5.186-2$ & $2.452-2$ & $8.010-3$ & $7.875-2$ & $3.642-2$ & $1.196-2$ \\
\hline 3 & 14 & 0.069 & 0.034 & 0.016 & $6.553-2$ & $3.666-2$ & $1.323-2$ & $9.052-2$ & $4.928-2$ & $2.107-2$ \\
\hline 3 & 15 & 0.023 & 0.010 & 0.003 & $7.676-2$ & $3.946-2$ & $1.414-2$ & $1.522-2$ & $6.707-3$ & $2.063-3$ \\
\hline 3 & 16 & 0.046 & 0.020 & 0.007 & $5.784-2$ & $3.379-2$ & $1.321-2$ & $4.264-2$ & $2.001-2$ & $6.523-3$ \\
\hline 3 & 17 & 0.055 & 0.026 & 0.012 & $8.582-2$ & $4.626-2$ & $1.927-2$ & $1.141-1$ & $5.648-2$ & $2.378-2$ \\
\hline 3 & 18 & 0.036 & 0.014 & 0.005 & $8.799-2$ & $4.168-2$ & $1.433-2$ & $7.205-2$ & $3.081-2$ & $9.763-3$ \\
\hline 3 & 19 & 0.105 & 0.050 & 0.023 & $9.557-2$ & $5.065-2$ & $2.401-2$ & $1.121-1$ & $5.432-2$ & $2.078-2$ \\
\hline 3 & 20 & & & & $7.761-2$ & $3.980-2$ & $1.469-2$ & $1.031-1$ & $4.112-2$ & $1.391-2$ \\
\hline 3 & 21 & & & & $7.417-2$ & $3.427-2$ & $1.175-2$ & $5.712-2$ & $2.624-2$ & $8.977-3$ \\
\hline 3 & 22 & 0.035 & 0.014 & 0.005 & $6.696-2$ & $3.221-2$ & $1.028-2$ & $5.259-2$ & $2.188-2$ & $6.572-3$ \\
\hline 3 & 23 & 0.139 & 0.045 & 0.013 & $4.850-2$ & $2.454-2$ & $8.389-3$ & $5.118-2$ & $2.366-2$ & $7.633-3$ \\
\hline 3 & 24 & 0.188 & 0.058 & 0.016 & $4.806-2$ & $2.592-2$ & $9.892-3$ & $4.211-2$ & $1.795-2$ & $5.938-3$ \\
\hline 3 & 25 & 0.077 & 0.035 & 0.020 & $4.152-2$ & $2.820-2$ & $1.836-2$ & $6.774-2$ & $3.585-2$ & $2.196-2$ \\
\hline 3 & 26 & 0.090 & 0.046 & 0.030 & $5.213-2$ & $3.718-2$ & $2.734-2$ & $6.841-2$ & $4.240-2$ & $3.173-2$ \\
\hline 3 & 27 & 0.087 & 0.039 & 0.015 & $2.772-1$ & $1.819-1$ & $6.321-2$ & $6.568-2$ & $2.808-2$ & $1.056-2$ \\
\hline 3 & 28 & 0.149 & 0.069 & 0.023 & $1.967-1$ & $1.057-1$ & $3.508-2$ & $1.574-1$ & $6.616-2$ & $2.190-2$ \\
\hline 3 & 29 & 0.081 & 0.037 & 0.012 & $1.179-1$ & $6.294-2$ & $2.079-2$ & $9.328-2$ & $3.978-2$ & $1.293-2$ \\
\hline 3 & 30 & 0.203 & 0.092 & 0.034 & $1.712-1$ & $7.721-2$ & $2.835-2$ & $2.324-1$ & $9.731-2$ & $3.474-2$ \\
\hline 3 & 31 & 0.138 & 0.060 & 0.023 & $1.199-1$ & $5.453-2$ & $2.051-2$ & $1.166-1$ & $5.706-2$ & $2.243-2$ \\
\hline
\end{tabular}

has not reported his results for oscillator strengths and/or the radiative rates. On the other hand, the corresponding results of PB for the allowed transitions, such as $1-3$ and 2-3, are lower. This is in spite of the fact that the $f$-values for both of these transitions are comparable in all three calculations. A closer comparison of our Figs. 6 and 7 with the corresponding figures of SST shows that the background $\Omega$ are also comparable, whereas those of PB (shown in their Fig. 2 for the same transitions) are lower by $\sim 30 \%$. This is because their values of $\Omega$ have not converged at lower energies, as explained in Sect. 3. For the same reason, the discrepancy between their results and ours or those of SST increases with increasing temperature, because the PB $\Upsilon$ values decrease with increasing temperature for (almost) all transitions, including the allowed ones. 


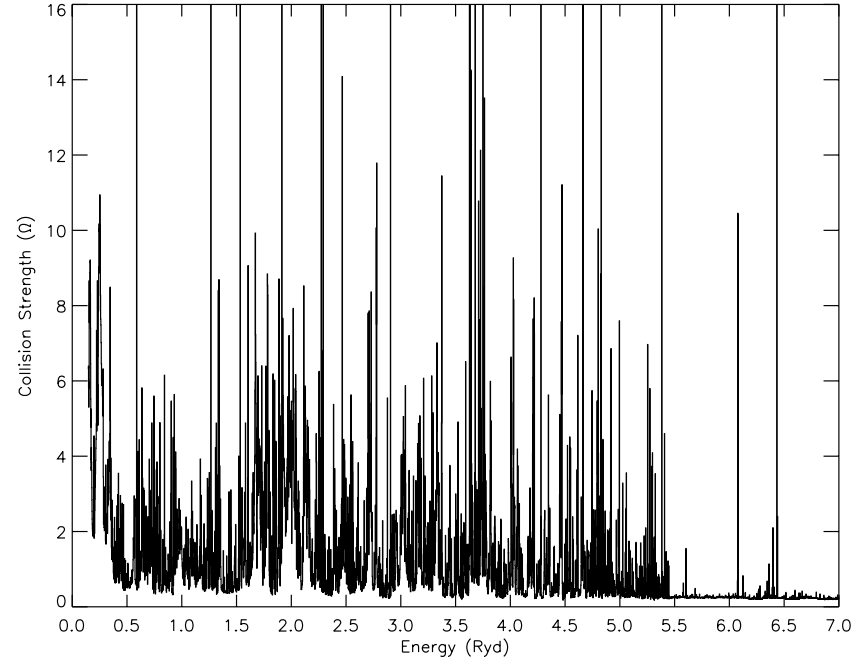

Fig. 5. Collision strengths for the $\left(3 \mathrm{~s}^{2} 3 \mathrm{p}^{5}\right)^{2} \mathrm{P}_{3 / 2}^{\circ}-{ }^{2} \mathrm{P}_{1 / 2}^{\circ}(1-2)$ transition of Fe X.

This limitation to their work becomes more apparent for some of the strong allowed transitions, such as 1-27, 1-28, 1-30, 2-29 and 2-31. For all of these transitions, the $\Upsilon$ results of PB remain nearly constant (within 10\%) in the temperature range of $5.4 \leq \log T_{\mathrm{e}} \leq 6.6 \mathrm{~K}$, whereas those of us or SST increase by $\sim 50 \%$ in the same range of temperature. Since the temperature of maximum $\mathrm{Fe} \mathrm{X}$ abundance in ionization equilibrium is around $10^{6} \mathrm{~K}$, the temperature range discussed above is highly important. It is clear from the comparisons shown in Table 6 that the $\Upsilon$ results of PB are underestimated by up to a factor of two, mostly for the allowed transitions, such as 1-3 and 2-3. Their corresponding results for some of the forbidden transitions are also underestimated, particularly towards the higher end of the temperature range, and examples of these are the $1-21,2-20,3-4$ and 3-26 transitions. It may also be noted that the temperature of $\sim 10^{6} \mathrm{~K}$ is only important in solar plasmas, and higher temperatures (of the order of $10^{7} \mathrm{~K}$ ) may be required for diagnostics of fusion plasmas. At such high temperatures, the $\Upsilon$ results of PB are clearly underestimated, and are likely to be unsuitable for applications.

Finally, in Figs. 8-11 we show resonances in $\Omega$ for the $1-4$, $1-5,3-4$ and 3-5 transitions, because similar resonances have also been shown by PB in their Figs. 3 and 4. It is interesting to note two clear differences for all the 4 transitions. Firstly, close to the thresholds the background values of $\Omega$ are higher in the PB work than in our calculations. This is perhaps a direct consequence of the difference in the coupling schemes adopted in the two calculations. Secondly, at energies above $\sim 5 \mathrm{Ryd}$ there is a dense group of resonances in the calculations of $\mathrm{PB}$, which is missing from our work. This is most likely because of the inclusion of all the 141 levels of the $3 s^{2} 3 p^{3} 3 d^{2}$ configuration in the PB work (for a limited range of partial waves only), whereas only 36 of these levels are included in our calculations, as stated in Sect. 2. Due to the limitations of our computational resources, a larger calculation with all the levels could not be performed at present, but it clearly emphasizes the need for including the additional levels. Nevertheless, in spite of the higher $\Omega$ background at lower energies and denser resonances

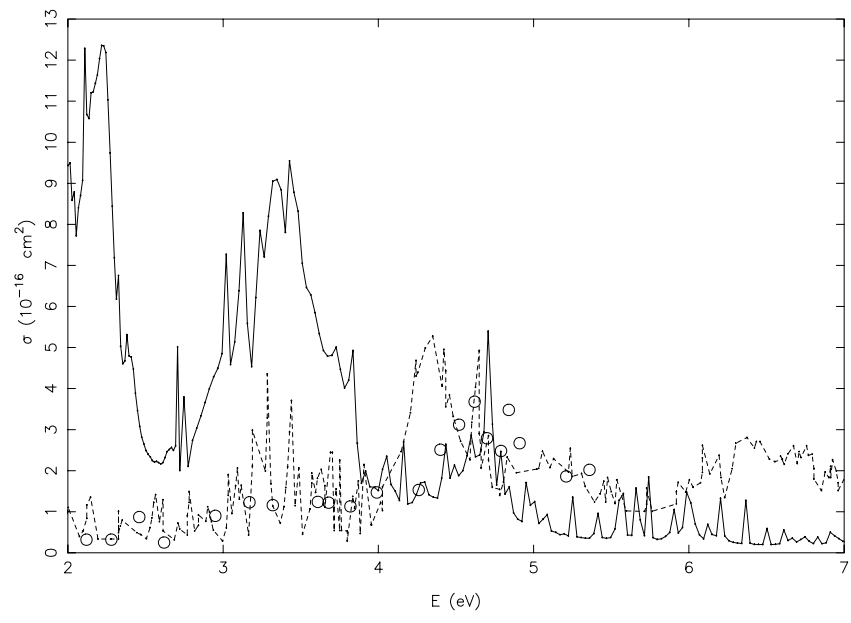

Fig. 12. Cross sections for the $\left(2 \mathrm{~s}^{2} 3 \mathrm{p}^{5}\right){ }^{2} \mathrm{P}_{3 / 2}^{\circ}-{ }^{2} \mathrm{P}_{1 / 2}^{\circ}(1-2)$ transition of $\mathrm{Fe} \mathrm{X}$. Continuous curve - present results, broken curve - earlier theoretical results of Tayal (2000), circles - experimental results of Niimura et al. (2002).

in the wider energy range, the $\Upsilon$ values of PB are still lower than ours. This is because of the denser and higher resonances we observe at energies close to the threshold, which have arisen due to the inclusion of fine-structure in the definition of channel coupling.

\section{Comparison with experiments}

$\mathrm{Fe} \mathrm{X}$ is one of the rare ions for which some experimental results are available for comparison for many atomic parameters, namely energy levels, life-times, cross sections and excitation rates. In our earlier work (Aggarwal \& Keenan 2004b), we made detailed comparisons and discussions for the first two parameters, and here we discuss corresponding experimental results for the other two. In Fig. 12 we show our results of cross sections $\sigma$ (in units of $\mathrm{cm}^{2}$ ) along with those of Tayal (2000) and Niimura et al. (2002) for the $\left(3 \mathrm{~s}^{2} 3 \mathrm{p}^{5}\right){ }^{2} \mathrm{P}_{3 / 2}^{\circ}-{ }^{2} \mathrm{P}_{1 / 2}^{\circ}$ (1-2) transition, in a limited energy range below $7 \mathrm{eV}$. There appears to be a better agreement between the theoretical results of Tayal and the experimental ones of Niimura et al., but the agreement with our present results is poor, especially at energies below $4 \mathrm{eV}$. The enhanced values of $\sigma$ we observe at low energies are not seen in the corresponding data of Tayal or Niimura et al., and the discrepancy cannot be resolved with the $18 \%$ error estimates of the experimental data. However, it will be unfair to assess the accuracy of either the theoretical or experimental data based on this limited comparison. On the experimental side, a few more measurements, especially at higher energies, will be helpful to make meaningful comparison with theory. On the theoretical side, separate calculations need to be performed keeping the very low energy range in mind. Nevertheless, we would like to emphasize here that since our calculations are oriented more towards the higher energy/temperature range in view of their applications to solar, astrophysical and fusion plasmas, the discrepancy observed with the experimental $\sigma$ results will not affect the accuracy of our results for $\Omega$ and $\Upsilon$. 
Table 7. Comparison of excitation rates $\left(10^{-9} \mathrm{~cm}^{3} \mathrm{~s}^{-1}\right)$ for some transitions from the ground state to higher excited levels of Fe X.

\begin{tabular}{lcccccccccc}
\hline \hline Level & Index & \multicolumn{3}{c}{ Wang \& Griem $(1983)$} & \multicolumn{3}{c}{ Mason (1975) } & \multicolumn{3}{c}{ Mann (1983) } \\
\hline Temperature $(\mathrm{eV})$ & 45 & 75 & 95 & 45 & 75 & 95 & 45 & 75 & 95 \\
\hline $3 \mathrm{p}^{4}\left({ }^{1} \mathrm{D}\right) 3 \mathrm{~d}^{2} \mathrm{~S}_{1 / 2}$ & 27 & 2.0 & 2.4 & 3.3 & 1.4 & 2.0 & 2.2 & 1.5 & 2.3 & 2.6 \\
$3 \mathrm{p}^{4}\left({ }^{3} \mathrm{P}\right) 3 \mathrm{~d}^{2} \mathrm{P}_{3 / 2}$ & 28 & 2.3 & 2.6 & 3.0 & 1.8 & 2.6 & 2.8 & 2.0 & 3.1 & 3.4 \\
$3 \mathrm{p}^{4}\left({ }^{3} \mathrm{P}\right) 3 \mathrm{~d}^{2} \mathrm{P}_{1 / 2}$ & 29 & .96 & 1.2 & 2.2 & .93 & 1.4 & 1.5 & 1.0 & 1.6 & 1.7 \\
$3 \mathrm{p}^{4}\left({ }^{3} \mathrm{P}\right) 3 \mathrm{~d}^{2} \mathrm{D}_{5 / 2}$ & 30 & 3.9 & 4.8 & 6.6 & 3.2 & 4.8 & 5.2 & 3.8 & 5.8 & 6.4 \\
$3 \mathrm{p}^{4}\left({ }^{3} \mathrm{P}\right) 3 \mathrm{~d}^{2} \mathrm{D}_{3 / 2}$ & 31 & 2.3 & 2.8 & 4.2 & 2.2 & 3.1 & 3.4 & 2.5 & 3.8 & 4.2 \\
\hline Level & Index & \multicolumn{3}{c}{ Tayal $(2001)$} & Pelan \& Berrington $(2001)$ & Present results \\
\hline Temperature $(\mathrm{eV})$ & 45 & 75 & 95 & 45 & 75 & 95 & 45 & 75 & 95 \\
\hline $3 \mathrm{p}^{4}\left({ }^{1} \mathrm{D}\right) 3 \mathrm{~d}^{2} \mathrm{~S}_{1 / 2}$ & 27 & 1.08 & 1.64 & 1.83 & 1.14 & 1.63 & 1.76 & 1.17 & 1.74 & 1.92 \\
$3 \mathrm{p}^{4}\left({ }^{3} \mathrm{P}\right) 3 \mathrm{~d}^{2} \mathrm{P}_{3 / 2}$ & 28 & 1.49 & 2.31 & 2.66 & 1.62 & 2.40 & 2.69 & 1.69 & 2.55 & 2.91 \\
$3 \mathrm{p}^{4}\left({ }^{3} \mathrm{P}\right) 3 \mathrm{~d}^{2} \mathrm{P}_{1 / 2}$ & 29 & .775 & 1.18 & 1.33 & .804 & 1.20 & 1.30 & .833 & 1.27 & 1.42 \\
$3 \mathrm{p}^{4}\left({ }^{3} \mathrm{P}\right) 3 \mathrm{~d}^{2} \mathrm{D}_{5 / 2}$ & 30 & 2.39 & 3.79 & 4.26 & 2.74 & 4.10 & 4.49 & 2.69 & 4.19 & 4.70 \\
$3 \mathrm{p}^{4}\left({ }^{3} \mathrm{P}\right) 3 \mathrm{~d}^{2} \mathrm{D}_{3 / 2}$ & 31 & 1.67 & 2.61 & 2.95 & 1.83 & 2.75 & 3.02 & 1.78 & 2.78 & 3.15 \\
\hline
\end{tabular}

Wang \& Griem (1983) have measured statistically averaged excitation rates from the ground state $2 \mathrm{p}^{5}{ }^{2} \mathrm{P}^{\circ}$ to higher excited levels of the $2 \mathrm{p}^{4} 3 \mathrm{~d}$ configuration, at three electron temperatures of 45, 75 and $95 \mathrm{eV}$. Their results are compared in Table 7 with our corresponding theoretical rates. Also included in this table are the earlier results from the work of Mason (1975) and Mann (1983). Both of them have adopted the DW code for the computations of $\Omega$, have employed limited $\mathrm{CI}$ in the generation of wavefunctions, and have not resolved resonances in thresholds region. As a result of these limitations, their rates are not only comparable with each other (within 20\%), but also agree with the measurements. However, later calculations of Pelan \& Berrington (2001) and Tayal (2001) are comparatively more sophisticated as already discussed in Sect. 1. The excitation rates obtained from their $\Upsilon$ values are also listed in Table 7 to provide a ready comparison. Both sets of these rates compare excellently with each other, at all temperatures and for all transitions listed in Table 7, and also agree within 10\% with our present results. However, in comparison to all the three $R$-matrix calculations, which employ better wavefunctions and include resonances in the determination of rates (or $\Upsilon$ ), the experimental results of Wang \& Griem are clearly overestimated by up to $50 \%$ for almost all transitions and at all temperatures. Nevertheless, their estimated errors are up to $60 \%$, depending on the transition and the temperature of measurement. Taking these error estimates into consideration, we may state that theory and experiment agree well at high temperatures.

\section{Conclusions}

In the present work, collision strengths and effective collision strengths for transitions among 90 fine-structure levels of $\mathrm{Fe} X$ are computed using the fully relativistic DARC code of Norrington \& Grant (2005). Results for both of these parameters are reported over a wide range of energy/temperature, suitable for applications in a variety of plasmas. Together with our earlier results for energy levels and radiative rates (Aggarwal \& Keenan 2004b), the present work reports a consistent set of results for many atomic parameters, and for a wider range of levels/transitions than hitherto available in the literature. Additionally, the present work is a clear improvement over the earlier results of BD, SST and PB. This has become possible due to the availability of better computational resources to us, which were perhaps not available to earlier workers. In comparison to the work of $\mathrm{BD}$, our calculations have significantly improved the accuracy of energy levels, radiative rates and collision strengths, by including extensive $\mathrm{CI}$ and performing the calculations in the $j j$ coupling. Furthermore, the levels/transitions range of their work has been increased by almost a factor of two, and the energy range by over a factor of four. In comparison to the work of SST, an overall improvement has been made by: (i) including additional $\mathrm{CI}$ in the generation of wavefunctions, and thus improving the accuracy of energy levels; (ii) extending the range of levels from 54 to 90 , and hence including many of the desired levels among which the transitions have already been observed; (iii) improving the accuracy of $\Omega$ values, by extending the range of partial waves (from 20 to 39) and the energy range (from $90 \mathrm{Ryd}$ to 210 Ryd); (iv) improving the $\Upsilon$ values by resolving resonances in a finer energy mesh and by including additional resonances; (v) performing the calculations in the $j j$ coupling instead of the SST semi-relativistic approach in the LSJ coupling scheme; and finally (vi) reporting the results for all transitions, instead of only a few. Similarly, the present work is an improvement over the work of PB mainly by extending the range of levels (transitions) from 31 (465) to 90 (4005), and by achieving convergence in values of $\Omega$ at all energies. The latter factor in particular has shown that the earlier reported results of $\Upsilon$ by PB are underestimated up to a factor of two, for many transitions and in a wider range of temperature.

Based on the available experimental data for energy levels and life-times, and on comparisons with several sets of calculations, we assessed the accuracy of our energy levels and radiative rates to be better than $3 \%$ and 20\%, respectively (Aggarwal \& Keenan 2004b). Experimental values of collision cross sections are confined to a single transition (1-2), and that too for a 
very limited energy range below $6 \mathrm{eV}$. Unfortunately, our theoretical results disagree with the experimental ones for this limited data. However, statistically averaged excitation rates are available from the plasma experiments for a few levels, and at three temperatures of 45, 75 and $95 \mathrm{eV}$. For these common levels there is satisfactory agreement among various sophisticated theoretical results, but the experimental values are assessed to be overestimated by up to $50 \%$. Nevertheless, this overestimation of experimental data are within the estimated error limits. Furthermore, based on several comparisons made with the earlier available results and our experience of similar calculations on a wide range of ions, we estimate the accuracy of our $\Omega$ and $\Upsilon$ data to be better than $20 \%$ for a majority of transitions. However, due to the presence of dense resonances in the threshold region, the results for $\Upsilon$ become more variable particularly towards the lower end of the temperature range. This particularly affects those transitions for which near-threshold resonances are dense as well as high. Similarly, radiative damping may reduce the resonance effect, which needs to be explored in future work. Furthermore, scope also remains for an improvement for transitions with higher upper levels, by including resonances from the higher lying levels of the neglected configurations, as highlighted in Sect. 4. However, this will significantly increase the range of levels and the calculations for which may be possible in future. Until then the present set of results for a range of atomic parameters should be highly useful for diagnostics and modelling applications.

Finally, it is unfortunate that in a recent elaborate "benchmarking" exercise Del Zanna et al. (2004) have adopted the atomic data of Pelan \& Berrington (2001), which have been shown to be deficient. Without performing a calculation it is difficult to assess the impact of our data on their derived results of wavelengths and intensities for many transitions of importance. The five brightest lines of Fe X (in decreasing order of intensity) are from the 1-30, 1-2, 1-28, 1-27 and 1-5 transitions - see Table 7 of Del Zanna et al. Only for the 1-2 transition, do our and the PB values of $\Upsilon$ agree closely in the entire temperature range (see Table 6 ). Their $\Upsilon$ results for the $1-27$, $1-28$ and 1-30 transitions are lower by up to $30 \%$, particularly towards the higher end of the temperature range, whereas their results for the 1-5 transition are lower between a factor of 4 and 2 , depending on the temperature. Therefore, it will be interesting to see the effect of our new data in a fresh calculation. We hope to perform such an exercise in the near future.

Acknowledgements. This work has been financed by the Engineering and Physical Sciences and Particle Physics and Astronomy Research Councils of the UK. We would like to thank Dr. Patrick Norrington for providing his DARC code prior to publication, and FPK is grateful to AWE Aldermaston for the award of a William Penny Fellowship. Finally, we thank an anonymous referee whose careful reading of the earlier version and constructive criticism have been helpful in improving the overall presentation of our work.

\section{References}

Aggarwal, K. M., \& Keenan, F. P. 2001, Phys. Scr., 64, 439

Aggarwal, K. M., \& Keenan, F. P. 2003a, MNRAS, 338, 412

Aggarwal, K. M., \& Keenan, F. P. 2003b, A\&A, 399, 799

Aggarwal, K. M., \& Keenan, F. P. 2004a, A\&A, 418, 371

Aggarwal, K. M., \& Keenan, F. P. 2004b, A\&A, 427, 763

Aggarwal, K. M., \& Keenan, F. P. 2005, A\&A, 429, 1117

Aggarwal, K. M., Deb, N. C., Keenan, F. P., \& Msezane, A. Z. 2000, J. Phys. B, 33, L391

Aggarwal, K. M., Keenan, F. P., \& Msezane, A. Z. 2001, J. Phys. B, 34, L757

Aggarwal, K. M., Keenan, F. P., \& Msezane, A. Z. 2003a, A\&A, 410, 349

Aggarwal, K. M., Keenan, F. P., \& Msezane, A. Z. 2003b, ApJS, 144, 169

Aggarwal, K. M., Keenan, F. P., \& Kisielius, R. 2004, A\&A, 420, 783

Berrington, K. A., Eissner, W. B., \& Norrington, P. H. 1995, Comput. Phys. Commun., 92, 290

Bhatia, A. K., \& Doschek, G. A. 1995, At. Data Nucl. Data Tables, 60, 97

Brosius, J. W., Davila, J. M., \& Thomas, R. J. 1998, ApJS, 119, 225

Chidichimo, M. C., Zemen, V., Tully, J. A., \& Berrington, K. A. 1999, A\&AS, 137, 175

Chidichimo, M. C., Del Zanna, G., Mason, H. E., et al. 2005, A\&A, 430,331

Deb, N. C., Gupta, G. P., \& Msezane, A. Z. 2002, ApJS, 141, 247

Del Zanna, G., Berrington, K. A., \& Mason, H. E. 2004, A\&A, 422, 731

Drake, J. J., Laming, J. M., \& Widing, K. G. 1995a, ApJ, 443, 393

Drake, J. J., Laming, J. M., \& Widing, K. G. 1995b, ApJ, 443, 416

Dyall, K. G., Grant, I. P., Johnson, C. T., Parpia, F. A., \& Plummer, E. P. 1989, Comput. Phys. Commun., 55, 424

Eissner, W., \& Seaton, M. J. 1972, J. Phys. B, 5, 2187

Eissner, W., Jones, M., \& Nussbaumer, H. 1974, Comput. Phys. Commun., 8, 270

Eissner, W., Galavis, M. E., Mendoza, C., \& Zeippen, C. J. 1999, A\&AS, 137, 165

Foster, V. J., Mathioudakis, M., Keenan, F. P., Drake, J. J., \& Widing, K. G. 1996, ApJ, 473, 560

Hibbert, A. 1975, Comput. Phys. Commun., 9, 141

Jonauskas, V., Keenan, F. P., Foord, M. E., et al. 2004, A\&A, 416, 383

Mann, J. B. 1983, At. Data Nucl. Data Tables, 29, 407

Mason, H. E. 1975, MNRAS, 170, 651

McKeown, K., Aggarwal, K. M., \& Keenan, F. P. 2004, Phys. Scr., 70, 295

Niimura, M., Cadez, I., Smith, S. J., \& Chutjian, A. 2002, Phys. Rev. Lett., 88, 103201

Norrington, P. H., \& Grant, I. P. 2005, Comput. Phys. Commun., in preparation

Pelan, J. C., \& Berrington, K. A. 2001, A\&A, 365, 258

Saraph, H. E. 1972, Comput. Phys. Commun., 3, 256

Tayal, S. S. 2000, ApJ, 544, 575

Tayal, S. S. 2001, ApJS, 132, 117

Wang, J. S., \& Griem, H. R. 1983, Phys. Rev., A27, 2249 


\section{Online Material}


K. M. Aggarwal and F. P. Keenan: Effective collision strengths for Fe X, Online Material p 2

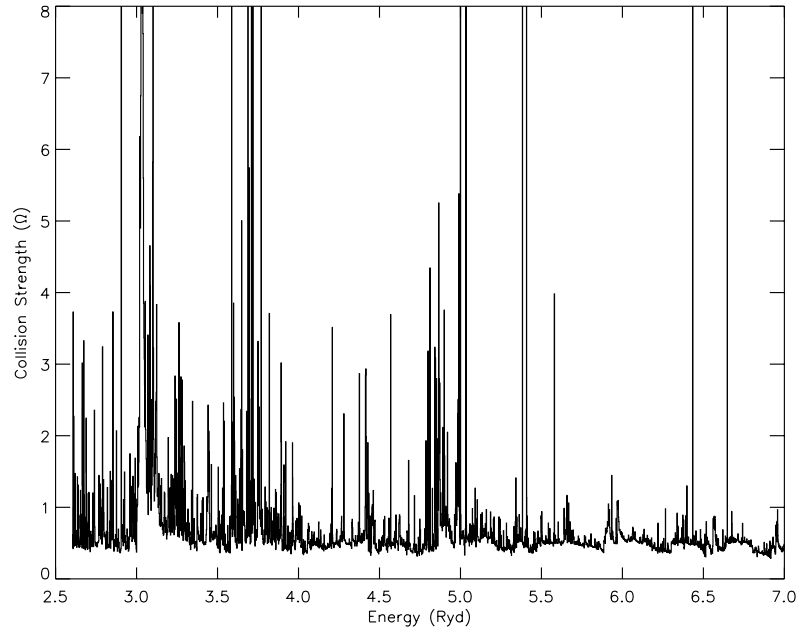

Fig. 6. Collision strengths for the $3 s^{2} 3 p^{5}{ }^{2} P_{3 / 2}^{o}-3 s 3 p^{6}{ }^{2} S_{1 / 2} \quad(1-3)$ transition of Fe X.

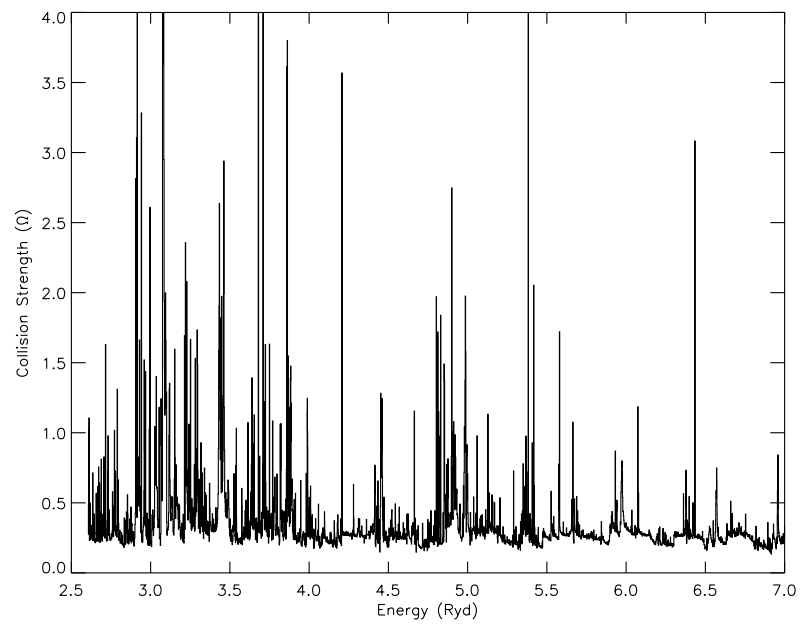

Fig. 7. Collision strengths for the $3 s^{2} 3 p^{5}{ }^{2} P_{1 / 2}^{o}-3 s 3 p^{6}{ }^{2} S_{1 / 2}(2-3)$ transition of $\mathrm{Fe} \mathrm{X}$.

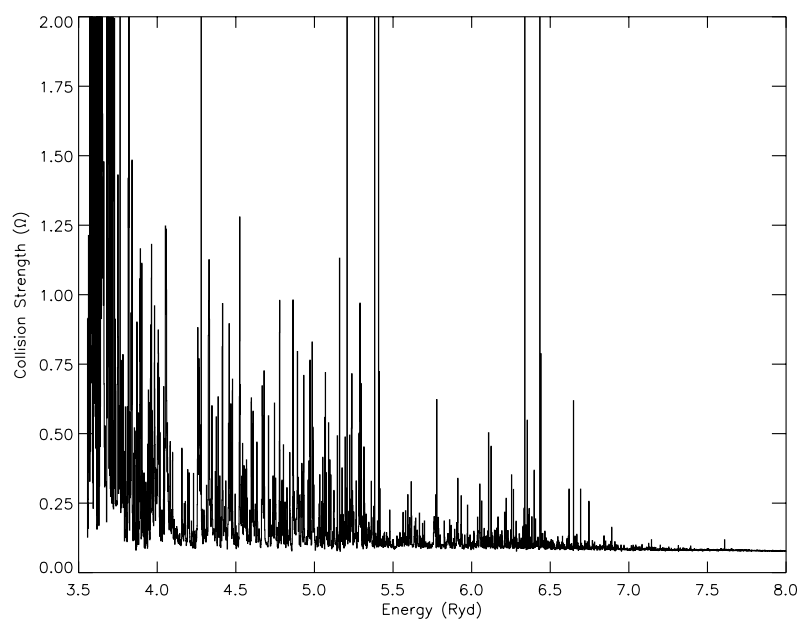

Fig. 8. Collision strengths for the $3 s^{2} 3 p^{5}{ }^{2} P_{3 / 2}^{o}-3 s^{2} 3 p^{4}\left({ }^{3} P\right) 3 d{ }^{4} D_{5 / 2}$ (1-4) transition of Fe X.

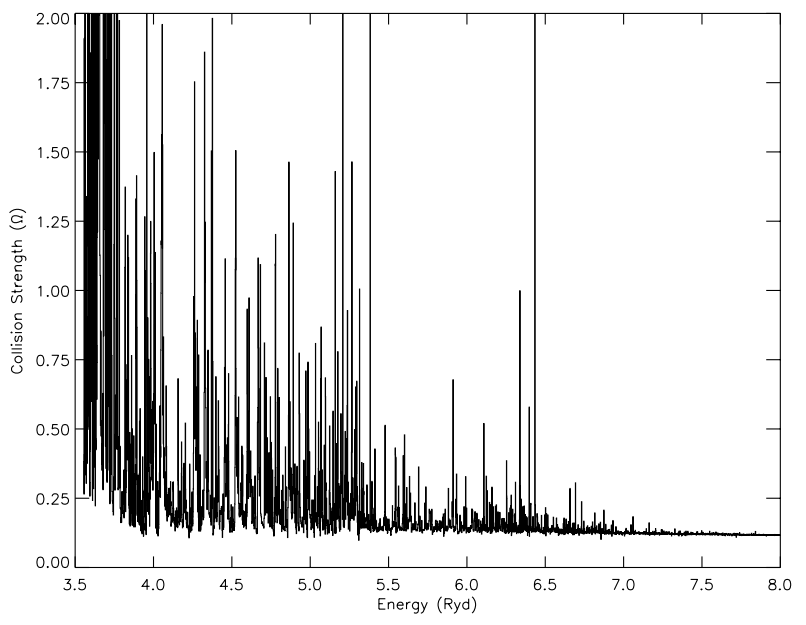

Fig. 9. Collision strengths for the $3 s^{2} 3 p^{5}{ }^{2} P_{3 / 2}^{o}-3 s^{2} 3 p^{4}\left({ }^{3} \mathrm{P}\right) 3 d{ }^{4} \mathrm{D}_{7 / 2}$ (1-5) transition of Fe X.

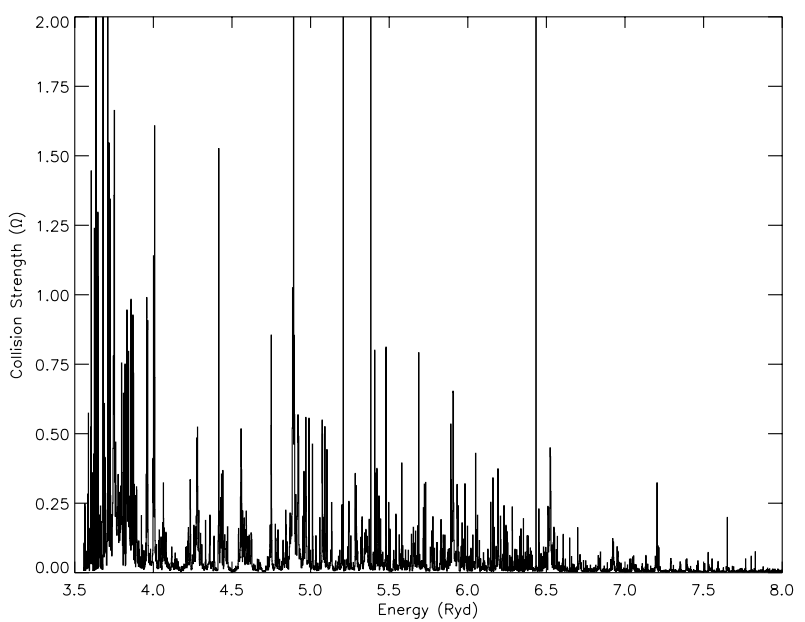

Fig. 10. Collision strengths for the $3 s 3 p^{6}{ }^{2} S_{1 / 2}-3 s^{2} 3 p^{4}\left({ }^{3} P\right) 3 d{ }^{4} D_{5 / 2}$ (3-4) transition of Fe X.

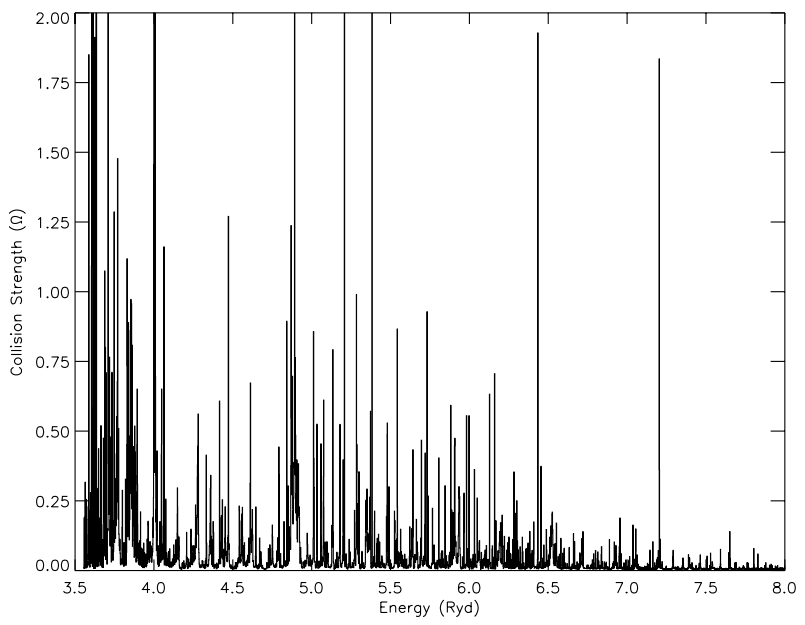

Fig. 11. Collision strengths for the $3 s 3 p^{6}{ }^{2} S_{1 / 2}-3 s^{2} 3 p^{4}\left({ }^{3} P\right) 3 d{ }^{4} D_{7 / 2}$ (3-5) transition of Fe X. 